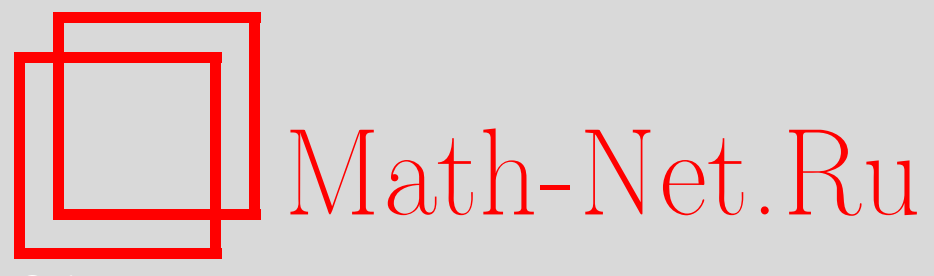

Г. Г. Брайчев, Наименьший тип целой функции с корнями заданных усредненных плотностей, расположенными на лучах или в угле, Матем. сб., 2016, том 207, номер 2, 45-80

DOI: https://doi.org/10.4213/sm8483

Использование Общероссийского математического портала Math-Net.Ru подразумевает, что вы прочитали и согласны с пользовательским соглашением http://www . mathnet.ru/rus/agreement

Параметры загрузки:

IP: 35.173 .219 .12

26 апреля 2023 г., $17: 08: 44$

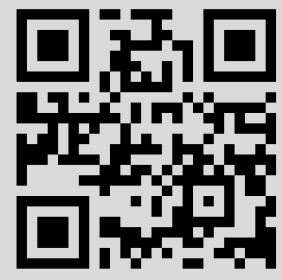




\title{
Г. Г. Брайчев
}

\section{Наименьший тип целой функции с корнями заданных усредненных плотностей, расположенными на лучах или в угле}

\begin{abstract}
Рассматривается задача о наименьшем возможном типе целых функций с корнями фиксированных верхней и нижней усредненных плотностей, лежащими на заданном множестве. В частности, дано решение этой задачи в нескольких важных случаях: все корни лежат в угле; между двумя прямыми; на лучах, разделяющих комплексную плоскость на равные углы.

Библиография: 15 названий.
\end{abstract}

Ключевые слова: тип целой функции, верхняя и нижняя усредненные плотности корней.

DOI: $10.4213 / \mathrm{sm} 8483$

\section{§ 1. Введение}

В классической теории целых функций и ее приложениях большую роль играет зависимость асимптотического поведения функции от распределения ее корней. Эта зависимость хорошо изучена в случае регулярного роста функции, отвечающего правильному распределению нулей (см., например, [1]). Потребности современной теории интерполяции и теории полноты различных систем в функциональных пространствах вызвали в последнее время повышенный интерес к изучению растущих нерегулярно целых функций конечного порядка с неизмеримой последовательностью нулей, расположенных на заданном множестве комплексной плоскости. Важнейшими примерами таких множеств являются прямая, луч, угол. Так, А. Ю. Попов в [2] дал новую оценку радиуса полноты систем экспонент, исследуя рост целых функций экспоненциального типа с вещественными симметричными нулями. Близкие вопросы изучались ранее в работе Р. Редхеффера [3]. Позднее в [4] был найден наименьший возможный тип при порядке $\rho \in(0,1)$ целых функций с положительными нулями заданной верхней плотности. В этом же классе целых функций в работе [5] В. Б. Шерстюковым определена величина наименьшего типа при условии, что задана не только верхняя, но и нижняя плотность последовательности нулей. Развивая эти результаты, в серии статей [6]-[8] автору удалось определить наименьшую величину типа, когда заданными являются усредненные верхняя и нижняя плотности. Тем самым задача о точной оценке снизу типа целой

Работа выполнена при поддержке Российского фонда фундаментальных исследований (грант № 13-01-00281). 
функции порядка $\rho \in(0,1)$ через классические плотности распределения ее нулей в случае, когда они лежат на луче, полностью исследована.

Следующим естественным шагом в данном направлении является изучение роста целых функций с нулями, расположенными не на одном, а на нескольких лучах или в угле. Совсем недавно А. Ю. Попов (см. [9]) определил наименьший возможный тип при порядке $\rho \in(0,1)$ целых функций с нулями заданной верхней плотности, расположенными в некотором угле, а В. Б. Шерстюков (см. [10]) решил такую же задачу с дополнительным условием на нижнюю плотность нулей. Автором [11] найдено наименьшее возможное значение типа при порядке $\rho \in(0, m)$ целых функций с нулями заданных верхней и нижней плотностей, расположенными на $m$ лучах, делящих плоскость на равные углы. Как и в случае целых функций с положительными нулями, актуальной является задача о точных оценках типа через интегральные характеристики распределения нулей - усредненные верхнюю и нижнюю плотности.

Настоящая работа является непосредственным продолжением исследований автора, начатых в [7], [8], [11], где можно найти более подробную историю вопроса и библиографию. Здесь мы определяем наименьшее значение типа целых функций с нулями, имеющими заданные верхнюю и нижнюю усредненные плотности. При этом предполагается, что нули лежат либо в некотором угле, либо между двумя прямыми, либо на нескольких правильно расположенных лучах, а также на некоторых более широких множествах. В частности, решена одна из проблем, поставленных в недавнем обзоре [9] (см. §1, задача $1^{*}$ ).

Введем необходимые понятия и определения. Типом целой функции $f(z)$ при порядке $\rho>0$ (коротко, $\rho$-типом) называется величина

$$
\sigma_{\rho}(f):=\varlimsup_{r \rightarrow+\infty} r^{-\rho} \ln \max _{|z|=r}|f(z)| .
$$

Пусть $\Lambda=\left(\lambda_{n}\right)_{n=1}^{\infty}-$ произвольная неограниченная последовательность комплексных чисел без конечных предельных точек. Поскольку отсутствие точки $\lambda=0$ в последовательности $\Lambda$ не влияет ни на одну из рассматриваемых здесь асимптотических характеристик, будем в дальнейшем считать, что $0 \notin \Lambda$. Обозначим через $n_{\Lambda}(t)=\sum_{\left|\lambda_{n}\right| \leqslant t} 1$ считающую (с учетом кратностей) функцию последовательности $\Lambda=\left(\lambda_{n}\right)_{n=1}^{\infty}$. Усредненной считающей функцией этой последовательности называют функцию

$$
N_{\Lambda}(r):=\int_{0}^{r} \frac{n_{\Lambda}(t)}{t} d t .
$$

Верхняя и нижняя $\rho$-плотности последовательности $\Lambda$ определяются равенствами

$$
\bar{\Delta}_{\rho}(\Lambda):=\varlimsup_{r \rightarrow+\infty} \frac{n_{\Lambda}(r)}{r^{\rho}}, \quad \Delta_{\rho}(\Lambda):=\varliminf_{r \rightarrow+\infty} \frac{n_{\Lambda}(r)}{r^{\rho}} .
$$

Аналогично определяются и усредненные верхняя и нижняя $\rho$-плотности $\Lambda$ :

$$
\bar{\Delta}_{\rho}^{*}(\Lambda):=\varlimsup_{r \rightarrow+\infty} \frac{N_{\Lambda}(r)}{r^{\rho}}, \quad \Delta_{\rho}^{*}(\Lambda):=\varliminf_{r \rightarrow+\infty} \frac{N_{\Lambda}(r)}{r^{\rho}} .
$$


Опишем структуру работы. В $\S 2,3$ исследуется случай, когда нули целой функции распределены в угле $\Gamma_{\theta}:=\{z \in \mathbb{C}:|\arg z| \leqslant \theta\}$ и обладают заданными усредненными $\rho$-плотностями. При этих условиях находится величина наименьшего возможного $\rho$-типа функции. Главным результатом этих параграфов является следующая теорема.

TeOpema 1. Пусть $\rho \in(0,1), \beta^{*}>0, \alpha^{*} \in\left[0, \beta^{*}\right], \theta \in[0, \pi / 2]$. Tun npu noрядке $\rho$ каждой целой функиии $f(z)$ с нулями $\Lambda_{f}$, лежащими в угле $\Gamma_{\theta}$ и имеющими усредненные $\rho$-плотности $\bar{\Delta}_{\rho}^{*}\left(\Lambda_{f}\right)=\beta^{*}, \Delta_{\rho}^{*}\left(\Lambda_{f}\right) \geqslant \alpha^{*}$, удовлетворяет неравенству

$$
\sigma_{\rho}(f) \geqslant \rho\left(\frac{\pi \alpha^{*} \cos \rho \theta}{\sin \pi \rho}+\max _{a>0} \int_{a a_{1}^{1 / \rho}}^{a a_{2}^{1 / \rho}} \frac{\left(\beta^{*} a^{-\rho}-\alpha^{*} \tau^{-\rho}\right)(\tau+\cos \theta)}{\tau^{2}+2 \tau \cos \theta+1} d \tau\right),
$$

где $a_{1}, a_{2}\left(0 \leqslant a_{1} \leqslant 1 \leqslant a_{2} \leqslant e\right)-$ корни уравнения

$$
a \ln \frac{e}{a}=\frac{\alpha^{*}}{\beta^{*}} .
$$

Равенство в оценке (1.1) достигается на некоторой целой функции с нулями $\tilde{\Lambda}$, лежащими на лучах $\gamma_{ \pm \theta}:=\{z \in \mathbb{C}: \arg z= \pm \theta\}$ и такими, что $\Delta_{\rho}^{*}(\widetilde{\Lambda})=\alpha^{*}, \bar{\Delta}_{\rho}^{*}(\widetilde{\Lambda})=\beta^{*}$.

Выделим несколько важных случаев теоремы 1. Полагая в ней $\alpha^{*}=0$, получаем следующий результат.

Теорема 2. Тип каждой целой функции $f(z)$ порядка $\rho \in(0,1)$ с нулями, расположенными в угле раствора $2 \theta \in[0, \pi]$ и имеющими верхнюю усредненную $\rho$-плотность $\beta^{*}$, удовлетворяет оценке

$$
\sigma_{\rho}(f) \geqslant \frac{\beta^{*} e \rho}{2} \max _{a>0} \frac{\ln \left(a^{2}+2 a \cos \theta+1\right)}{a^{\rho}} .
$$

Существует иелая функиия, нулями которой служит некоторая последовательность $\widetilde{\Lambda} \subset \gamma_{\theta} \cup \gamma_{-\theta}$ с верхней усредненной $\rho$-плотностью $\bar{\Delta}_{\rho}^{*}(\widetilde{\Lambda})=\beta^{*}$, реализующая равенство в этой оценке.

Говорят, что последовательность $\Lambda$ измерима, если $\bar{\Delta}_{\rho}^{*}(\Lambda)=\triangleq_{\rho}^{*}(\Lambda)$, а общее значение $\Delta_{\rho}^{*}(\Lambda):=\bar{\Delta}_{\rho}^{*}(\Lambda)=\underline{\Delta}_{\rho}^{*}(\Lambda)$ называют усредненной $\rho$-Плотностью $\Lambda$. Теперь из теоремы 1 при $\alpha^{*}=\beta^{*}$ для целых функций с измеримыми нулями вытекает такое утверждение.

Теорема 3. Тип каждой целой функции $f(z)$ порядка $\rho \in(0,1)$ с измеримыми нулями усредненной $\rho$-плотности $\beta^{*}$, расположенными в угле раствора $2 \theta \in[0, \pi]$, удовлетворяет оценке

$$
\sigma_{\rho}(f) \geqslant \beta^{*} \frac{\pi \rho \cos \rho \theta}{\sin \pi \rho} .
$$

Равенство реализуется на целой функиии, нулями которой служит измеримая последовательность $\widetilde{\Lambda} \subset \gamma_{\theta} \cup \gamma_{-\theta}$ с усредненной $\rho$-плотностью $\beta^{*} / 2$ на каждом из лучей $\gamma_{ \pm \theta}$. 
Если $\theta=0$, то нули функции расположены на положительной полуоси $\mathbb{R}_{+}$. В этом случае теорема 1 дает значение наименьшего $\rho$-типа целой функции с положительными нулями заданных усредненных $\rho$-плотностей, найденное ранее в работе [7] (см. также [8]):

при любых заданных $\rho \in(0,1), \beta^{*}>0, \alpha^{*} \in\left[0, \beta^{*}\right]$ экстремальная величина

$$
s^{*}\left(\alpha^{*}, \beta^{*}, \rho\right):=\inf \left\{\sigma_{\rho}(f): \Lambda_{f}=\Lambda \subset \mathbb{R}_{+}, \triangleq_{\rho}^{*}(\Lambda) \geqslant \alpha^{*}, \bar{\Delta}_{\rho}^{*}(\Lambda)=\beta^{*}\right\}
$$

вычисляется по формуле

$$
s^{*}\left(\alpha^{*}, \beta^{*}, \rho\right)=\rho\left(\frac{\pi \alpha^{*}}{\sin \pi \rho}+\max _{a>0} \int_{a a_{1}^{1 / \rho}}^{a a_{2}^{1 / \rho}} \frac{\left(\beta^{*} a^{-\rho}-\alpha^{*} \tau^{-\rho}\right)}{\tau+1} d \tau\right),
$$

где $a_{1}$ и $a_{2}$ по-прежнему являются корнями уравнения (1.2); нижняя грань $s^{*}\left(\alpha^{*}, \beta^{*}, \rho\right)$ достигается на некоторой последовательности $\widetilde{\Lambda} \subset \mathbb{R}_{+}$такой, чmo $\Delta_{\rho}^{*}(\widetilde{\Lambda})=\alpha^{*}$ u $\bar{\Delta}_{\rho}^{*}(\widetilde{\Lambda})=\beta^{*}$.

Дальнейшее содержание таково. В $\S 4$ мы усиливаем теорему 1 , показывая, что ее утверждение остается в силе для функций с нулями, лежащими в некоторых "близких" к углу криволинейных областях. В $\S 5$ решаются аналогичные задачи для целых функций с нулями, расположенными в областях, "близких" не к одному, а к нескольким непересекающимся углам, биссектрисы которых делят плоскость на равные части. Следующий $\S 6$ посвящен важным случаям расположения нулей целых функций порядка $\rho \in(0,2)$ на одной прямой, между двумя пересекающимися или параллельными прямыми, а также и в чуть более широких множествах. Здесь же даются точные оценки снизу для индикатрисы роста рассматриваемых целых функций. Наконец, в заключительном $\S 7$ устанавливаются некоторые свойства величины экстремального $\rho$-типа как функции соответствующих параметров.

\section{§ 2. Доказательство теоремы 1: основная оценка}

Пусть $\rho \in(0,1), \beta^{*}>0, \alpha^{*} \in\left[0, \beta^{*}\right], \theta \in[0, \pi / 2]-$ фиксированные числа, $a_{1}$ и $a_{2}-$ корни уравнения (1.2). Пусть далее $f(z)$ - целая функция, все нули которой $\Lambda=\Lambda_{f}$ лежат в угле $\Gamma_{\theta}=\{z \in \mathbb{C}:|\arg z| \leqslant \theta\}$ и имеют усредненные $\rho$-плотности

$$
\bar{\Delta}_{\rho}^{*}(\Lambda)=\beta^{*}, \quad \Delta_{\rho}^{*}(\Lambda) \geqslant \alpha^{*} .
$$

Наша ближайшая задача - доказать неравенство (1.1), связывающее $\rho$-тип $\sigma_{\rho}(f)$ такой функции с усредненными плотностями ее нулей.

Без ограничения общности будем в дальнейшем считать, что $f(0)=1$, и упорядочим нули функции $f(z)$ по возрастанию модуля, записав их в виде

$$
\lambda_{n}=r_{n} e^{i \theta_{n}}, \quad\left|\theta_{n}\right| \leqslant \theta, \quad r_{n}=\left|\lambda_{n}\right| \nearrow+\infty .
$$

По теореме Адамара (см. [1; гл. I, §10]) функция $f(z)$ представляется в виде канонического произведения

$$
f(z)=\prod_{n=1}^{\infty}\left(1-\frac{z}{\lambda_{n}}\right), \quad z \in \mathbb{C} .
$$


Рассмотрим вспомогательную целую функцию

$$
\begin{aligned}
F(z) & =f(z) \overline{f(\bar{z})}=\prod_{n=1}^{\infty}\left(1-\frac{z}{\lambda_{n}}\right)\left(1-\frac{z}{\bar{\lambda}_{n}}\right) \\
& =\prod_{n=1}^{\infty}\left(1-z\left(\frac{1}{\lambda_{n}}+\frac{1}{\bar{\lambda}_{n}}\right)+\left(\frac{z}{\left|\lambda_{n}\right|}\right)^{2}\right)=\prod_{n=1}^{\infty}\left(1-\frac{2 z}{r_{n}} \cos \theta_{n}+\left(\frac{z}{r_{n}}\right)^{2}\right)
\end{aligned}
$$

где черта означает комплексное сопряжение. Для любого $z \in \mathbb{C},|z|=r$, имеем

$$
\omega_{n}(z):=\left|1-\frac{2 z}{r_{n}} \cos \theta_{n}+\left(\frac{z}{r_{n}}\right)^{2}\right| \leqslant 1+\frac{2 r}{r_{n}} \cos \theta_{n}+\left(\frac{r}{r_{n}}\right)^{2}=\omega_{n}(-r) .
$$

Учитывая теперь, что для $\left|\theta_{n}\right| \leqslant \theta$ справедливо соотношение $\cos \theta_{n} \geqslant \cos \theta$, получаем

$$
\begin{aligned}
& \max _{|z|=r}|F(z)|=\prod_{n=1}^{\infty} \omega_{n}(-r) \geqslant \prod_{n=1}^{\infty}\left(1+\frac{2 r}{r_{n}} \cos \theta+\left(\frac{r}{r_{n}}\right)^{2}\right), \\
& \ln \max _{|z|=r}|F(z)| \geqslant \sum_{n=1}^{\infty} \ln \left(1+\frac{2 r}{r_{n}} \cos \theta+\left(\frac{r}{r_{n}}\right)^{2}\right)=: \sigma(r) .
\end{aligned}
$$

Заметим, что неравенство превращается в равенство, если $\left|\theta_{n}\right|=\theta$ для всех $n \in \mathbb{N}$. Положим временно для сокращения записи $b=\cos \theta$. Как и прежде, будем обозначать через $n(t)=n_{\Lambda}(t)$ считающую функцию нулей $f(z)$ и через $N(t)=N_{\Lambda}(t)$ - их усредненную считающую функцию. В дальнейшем зависимость от $\Lambda$ в обозначениях этих и других характеристик будем опускать там, где это не вызывает недоразумений.

Применяя стандартное интегрирование по частям в интеграле Стилтьеса, преобразуем выражение для $\sigma(r)$ :

$$
\begin{aligned}
\sigma(r) & =\int_{0}^{+\infty} \ln \left(1+\frac{2 r}{t} b+\left(\frac{r}{t}\right)^{2}\right) d n(t) \\
& =\left.\ln \left(1+\frac{2 r}{t} b+\left(\frac{r}{t}\right)^{2}\right) n(t)\right|_{0} ^{+\infty}-\int_{0}^{+\infty} \frac{-2 b r / t^{2}-2 r^{2} / t^{3}}{1+2 b r / t+(r / t)^{2}} n(t) d t \\
& =2 r \int_{0}^{+\infty} \frac{b t+r}{r^{2}+2 b r t+t^{2}} \underbrace{\frac{n(t) d t}{t}}_{d N(t)} \\
& =2 r\left(\left.\frac{b t+r}{r^{2}+2 b r t+t^{2}} N(t)\right|_{0} ^{+\infty}+\int_{0}^{+\infty} N(t) \frac{b r^{2}+2 r t+b t^{2}}{\left(r^{2}+2 b r t+t^{2}\right)^{2}} d t\right)
\end{aligned}
$$

Здесь обе подстановки обращаются в нуль. Действительно, из условия $f(0)=1$ следует, что $n(0)=N(0)=0$, а из конечности величины $\bar{\Delta}_{\rho}^{*}$ и известного (см. [1; гл. IV, §1]) неравенства

$$
\bar{\Delta}_{\rho} \leqslant e \rho \bar{\Delta}_{\rho}^{*}
$$


вытекает, что $N(t)=O\left(t^{\rho}\right)$ и $n(t)=O\left(t^{\rho}\right)$ при $t \rightarrow+\infty$. После замены переменной $t=r \tau$ в последнем интеграле получаем

$$
\sigma(r)=2 \int_{0}^{+\infty} N(r \tau) \frac{b \tau^{2}+2 \tau+b}{\left(\tau^{2}+2 b \tau+1\right)^{2}} d \tau=2 r^{\rho} \int_{0}^{+\infty} \frac{N(r \tau)}{(r \tau)^{\rho}} \frac{\tau^{\rho}\left(b \tau^{2}+2 \tau+b\right)}{\left(\tau^{2}+2 b \tau+1\right)^{2}} d \tau .
$$

Подводя итог вычислениям, можем записать соотношение

$$
\frac{\ln \max _{|z|=r}|F(z)|}{r^{\rho}} \geqslant \frac{\sigma(r)}{r^{\rho}}=2 \int_{0}^{+\infty} \Delta^{*}(r \tau) K(\tau) d \tau,
$$

где мы обозначили

$$
\Delta^{*}(r):=\frac{N(r)}{r^{\rho}}, \quad K(\tau):=\frac{\tau^{\rho}\left(b \tau^{2}+2 \tau+b\right)}{\left(\tau^{2}+2 b \tau+1\right)^{2}} .
$$

В случае, когда $\left|\theta_{n}\right|=\theta$ при всех $n \in \mathbb{N}$, выполняется равенство

$$
\frac{\ln \max _{|z|=r}|F(z)|}{r^{\rho}}=\frac{\sigma(r)}{r^{\rho}}=2 \int_{0}^{+\infty} \Delta^{*}(r \tau) K(\tau) d \tau .
$$

Оценим снизу функцию $\Delta^{*}(r)$.

Рассмотрим сначала случай, когда последовательность нулей целой функции неизмерима, т.е. $\Delta^{*}<\bar{\Delta}^{*}$. Из определения усредненной считающей функции $N(x)$ вытекает, что при всех положительных $r, \tau, a$ выполняется оценка

$$
N(r \tau)=N\left(\frac{r}{a}\right)+\int_{r a^{-1}}^{r \tau} \frac{n(x)}{x} d x \geqslant N\left(\frac{r}{a}\right)+n\left(\frac{r}{a}\right) \ln a \tau .
$$

Отсюда уже нетрудно получить, что

$$
\begin{aligned}
\Delta^{*}(r \tau) & \geqslant \frac{N(r / a)+n(r / a) \ln a \tau}{(r \tau)^{\rho}}=\frac{N(r / a)}{(r / a)^{\rho}} \frac{1+\nu(r / a) \ln a \tau}{(a \tau)^{\rho}} \\
& =\Delta^{*}(r / a) \frac{1+\nu(r / a) \ln a \tau}{(a \tau)^{\rho}}
\end{aligned}
$$

где обозначено $\nu(r):=n(r) / N(r)$. Далее нам потребуется одно вспомогательное утверждение, доказанное в [7].

ЛЕмма 2.1. Пусть $\Lambda$ - неизмеримая последовательность положительных чисел, т.е. $\Delta^{*}<\bar{\Delta}^{*}$. Тогда найдется последовательность $R_{j} \rightarrow+\infty$, накоторой выполняются соотношения

$$
\nu\left(R_{j}\right) \geqslant \rho, \quad \Delta^{*}\left(R_{j}\right) \rightarrow \bar{\Delta}^{*}, \quad j \rightarrow \infty .
$$

Зафиксируем произвольно положительные числа $a>0$ и $\varepsilon>0$. Из определения усредненной нижней $\rho$-плотности последовательности $\Lambda$ найдем число $c>0$ так, чтобы при каждом $r \geqslant a c a_{2}^{1 / \rho}$ для всех $\tau \geqslant c / r$ выполнялось неравенство $\Delta^{*}(r \tau) \geqslant \alpha_{\varepsilon}^{*}:=\alpha^{*} /(1+\varepsilon)$. Пусть $r$ пробегает значения $r_{j}=a R_{j}$, где 
$R_{j}$ взяты из леммы 2.1. Применяя эту лемму, для всех достаточно больших $j$, скажем $j \geqslant j_{0}$, получим

$$
\nu\left(R_{j}\right)=\nu\left(\frac{r_{j}}{a}\right) \geqslant \rho, \quad \Delta^{*}\left(R_{j}\right)=\Delta^{*}\left(\frac{r_{j}}{a}\right) \geqslant \beta_{\varepsilon}^{*}:=\frac{\beta^{*}}{1+\varepsilon} .
$$

Продолжая доказательство оценки (1.1), можем записать соотношение

$$
\Delta^{*}\left(r_{j} \tau\right) \geqslant \beta_{\varepsilon}^{*} \frac{1+\rho \ln a \tau}{(a \tau)^{\rho}}, \quad \tau \geqslant \frac{c}{r_{j}}, \quad j \geqslant j_{0} .
$$

Введем для $\tau>0$ вспомогательную функцию

$$
\psi_{r}(\tau)= \begin{cases}\beta_{\varepsilon}^{*} \frac{1+\rho \ln a \tau}{(a \tau)^{\rho}}, & \tau \in\left(\left(a a_{2}^{1 / \rho}\right)^{-1},\left(a a_{1}^{1 / \rho}\right)^{-1}\right), \\ \alpha_{\varepsilon}^{*}, & \tau \notin\left(\left(a a_{2}^{1 / \rho}\right)^{-1},\left(a a_{1}^{1 / \rho}\right)^{-1}\right) .\end{cases}
$$

Здесь и ниже $a_{1} \leqslant 1 \leqslant a_{2}$ суть корни уравнения (1.2). Обратим внимание на то, что при $\alpha^{*}=0$ имеем $a_{1}=0, a_{2}=e$, и функция $\psi_{r}(\tau)$ принимает вид

$$
\psi_{r}(\tau)= \begin{cases}\beta_{\varepsilon}^{*} \frac{1+\rho \ln a \tau}{(a \tau)^{\rho}}, & \tau \in\left(\left(a e^{1 / \rho}\right)^{-1},+\infty\right), \\ 0, & \tau \notin\left(\left(a e^{1 / \rho}\right)^{-1},+\infty\right) .\end{cases}
$$

Учитывая, что для $\tau \geqslant c r_{j}^{-1}$ выполняется $\Delta^{*}\left(r_{j} \tau\right) \geqslant \alpha_{\varepsilon}^{*}$, можем утверждать, что при всех $r_{j} \geqslant a c a_{2}^{1 / \rho}$ и $\tau \geqslant c r_{j}^{-1}, j \geqslant j_{0}$, справедливо неравенство

$$
\Delta^{*}\left(r_{j} \tau\right) \geqslant \psi_{r_{j}}(\tau)
$$

Последнее вместе с (2.3) во всех случаях влечет за собой оценку

$$
\frac{\ln \max _{|z|=r_{j}}|F(z)|}{r_{j}^{\rho}} \geqslant 2 \int_{c r_{j}^{-1}}^{+\infty} \psi_{r_{j}}(\tau) K(\tau) d \tau, \quad j \geqslant j_{0} .
$$

Интеграл в правой части преобразуем следующим образом:

$$
\begin{gathered}
\int_{c r_{j}^{-1}}^{+\infty} \psi_{r_{j}}(\tau) K(\tau) d \tau=\alpha_{\varepsilon}^{*}\left(\int_{c r_{j}^{-1}}^{\left(a a_{2}^{1 / \rho}\right)^{-1}} K(\tau) d \tau+\int_{\left(a a_{1}^{1 / \rho}\right)^{-1}}^{+\infty} K(\tau) d \tau\right) \\
\quad+\beta_{\varepsilon}^{*} \int_{\left(a a_{2}^{1 / \rho}\right)^{-1}}^{\left(a a_{1}^{1 / \rho}\right)^{-1}} \frac{1+\rho \ln a \tau}{(a \tau)^{\rho}} K(\tau) d \tau \\
=\alpha_{\varepsilon}^{*} \int_{c r_{j}^{-1}}^{+\infty} K(\tau) d \tau+\int_{\left(a a_{2}^{1 / \rho}\right)^{-1}}^{\left(a a_{1}^{1 / \rho}\right)^{-1}}\left(\beta_{\varepsilon}^{*} \frac{1+\rho \ln a \tau}{(a \tau)^{\rho}}-\alpha_{\varepsilon}^{*}\right) K(\tau) d \tau \\
=\alpha_{\varepsilon}^{*}\left(\int_{0}^{+\infty} K(\tau) d \tau-\int_{0}^{c r_{j}^{-1}} K(\tau) d \tau\right) \\
\quad+\int_{\left(a a_{2}^{1 / \rho}\right)^{-1}}^{\left(a a_{1}^{1 / \rho}\right)^{-1}} \frac{\left(\beta_{\varepsilon}^{*} a^{-\rho}(1+\rho \ln a \tau)-\alpha_{\varepsilon}^{*} \tau^{\rho}\right) K(\tau)}{\tau^{\rho}} d \tau .
\end{gathered}
$$


Переходя теперь в неравенстве (2.6) к пределу по $j \rightarrow \infty$, получаем

$$
\begin{aligned}
\frac{\sigma_{\rho}(F)}{2} & \geqslant \lim _{j \rightarrow \infty} \int_{c r_{j}^{-1}}^{+\infty} \psi_{r_{j}}(\tau) K(\tau) d \tau \\
& =\alpha_{\varepsilon}^{*} \int_{0}^{+\infty} K(\tau) d \tau+\int_{\left(a a_{2}^{1 / \rho}\right)^{-1}}^{\left(a a_{1}^{1 / \rho}\right)^{-1}} \frac{\left(\beta_{\varepsilon}^{*} a^{-\rho}(1+\rho \ln a \tau)-\alpha_{\varepsilon}^{*} \tau^{\rho}\right) K(\tau)}{\tau^{\rho}} d \tau
\end{aligned}
$$

Устремив здесь $\varepsilon$ к нулю, приходим к неравенству

$$
\frac{\sigma_{\rho}(F)}{2} \geqslant \alpha^{*} \underbrace{\int_{0}^{+\infty} K(\tau) d \tau}_{I_{1}}+\underbrace{\int_{\left(a a_{2}^{1 / \rho}\right)^{-1}}^{\left(a a_{1}^{1 / \rho}\right)^{-1}} \frac{\left(\beta^{*} a^{-\rho}(1+\rho \ln a \tau)-\alpha^{*} \tau^{\rho}\right) K(\tau)}{\tau^{\rho}} d \tau}_{I_{2}}
$$

Интегралы $I_{1}$ и $I_{2}$ в этой оценке возьмем по частям, используя равенство

$$
K(\tau)=-\tau^{\rho}\left(\frac{b \tau+1}{\tau^{2}+2 b \tau+1}\right)^{\prime}=\frac{\tau^{\rho}\left(b \tau^{2}+2 \tau+b\right)}{\left(\tau^{2}+2 b \tau+1\right)^{2}} .
$$

Для $I_{1}$ получим:

$$
\begin{gathered}
I_{1}=\int_{0}^{+\infty} K(\tau) d \tau=-\int_{0}^{+\infty} \tau^{\rho} d\left(\frac{b \tau+1}{\tau^{2}+2 b \tau+1}\right)=-\left.\tau^{\rho}\left(\frac{b \tau+1}{\tau^{2}+2 b \tau+1}\right)\right|_{0} ^{+\infty} \\
\quad+\rho \int_{0}^{+\infty} \tau^{\rho-1}\left(\frac{b \tau+1}{\tau^{2}+2 b \tau+1}\right) d \tau=\frac{\pi \rho}{\sin \pi \rho} \cos \rho \theta
\end{gathered}
$$

При вычислении последнего интеграла использовалась известная формула (см. [12; п. 2.2.9]). Таким образом,

$$
I_{1}=\frac{\pi \rho}{\sin \pi \rho} \cos \rho \theta
$$

Аналогично вычисляем интеграл $I_{2}$ :

$$
\begin{aligned}
I_{2}=- & \int_{\left(a a_{2}^{1 / \rho}\right)^{-1}}^{\left(a a_{1}^{1 / \rho}\right)^{-1}}\left(\beta^{*} a^{-\rho} \ln e(a \tau)^{\rho}-\alpha^{*} \tau^{\rho}\right) d\left(\frac{b \tau+1}{\tau^{2}+2 b \tau+1}\right) \\
=- & \left.\left(\frac{b \tau+1}{\tau^{2}+2 b \tau+1}\right)\left(\beta^{*} a^{-\rho} \ln e(a \tau)^{\rho}-\alpha^{*} \tau^{\rho}\right)\right|_{\left(a a_{2}^{1 / \rho}\right)^{-1}} ^{\left(a a_{1}^{1 / \rho}\right)^{-1}} \\
& +\rho \int_{\left(a a_{2}^{1 / \rho}\right)^{-1}}^{\left(a a_{1}^{1 / \rho}\right)^{-1}} \frac{\left(\beta^{*} a^{-\rho}-\alpha^{*} \tau^{\rho}\right)(b \tau+1)}{\tau^{2}+2 b \tau+1} \frac{d \tau}{\tau}
\end{aligned}
$$

Проверим, что внеинтегральное слагаемое равно нулю. Действительно,

$$
\beta^{*} a^{-\rho} \ln e(a \tau)^{\rho}-\alpha^{*} \tau^{\rho}=\beta^{*} \tau^{\rho}\left((a \tau)^{-\rho} \ln \frac{e}{(a \tau)^{-\rho}}-\frac{\alpha^{*}}{\beta^{*}}\right),
$$

а выражение в скобках при $\tau=\left(a a_{j}^{1 / \rho}\right)^{-1}$ дает $a_{j} \ln \left(e / a_{j}\right)-\alpha^{*} / \beta^{*}=0$, поскольку $a_{j}, j=1,2,-$ корни уравнения (1.2). Следовательно,

$$
I_{2}=\rho \int_{\left(a a_{2}^{1 / \rho}\right)^{-1}}^{\left(a a_{1}^{1 / \rho}\right)^{-1}} \frac{\left(\beta^{*} a^{-\rho}-\alpha^{*} \tau^{\rho}\right)(b \tau+1)}{\tau^{2}+2 b \tau+1} \frac{d \tau}{\tau}
$$


или, после замены переменной $t=\tau^{-1}$,

$$
I_{2}=\rho \int_{a a_{1}^{1 / \rho}}^{a a_{2}^{1 / \rho}} \frac{\left(\beta^{*} a^{-\rho}-\alpha^{*} t^{-\rho}\right)(t+b)}{t^{2}+2 b t+1} d t .
$$

Подводя итог последним преобразованиям, из (2.7)-(2.9) получаем

$$
\frac{\sigma_{\rho}(F)}{2} \geqslant \rho\left(\frac{\pi \alpha^{*}}{\sin \pi \rho} \cos \rho \theta+\int_{a a_{1}^{1 / \rho}}^{a a_{2}^{1 / \rho}} \frac{\left(\beta^{*} a^{-\rho}-\alpha^{*} t^{-\rho}\right)(t+b)}{t^{2}+2 b t+1} d t\right) .
$$

Заметим теперь, что

$$
M_{F}(r)=\max _{|z|=r}|F(z)|=\max _{|z|=r}|f(z)||\overline{f(\bar{z})}| \leqslant \max _{|z|=r}|f(z)|^{2}=M_{f}^{2}(r),
$$

откуда

$$
\sigma_{\rho}(F)=\varlimsup_{r \rightarrow+\infty} r^{-\rho} \ln M_{F}(r) \leqslant \varlimsup_{r \rightarrow+\infty} r^{-\rho} \ln M_{f}^{2}(r)=2 \sigma_{\rho}(f) .
$$

Таким образом,

$$
\sigma_{\rho}(f) \geqslant \frac{\sigma_{\rho}(F)}{2} \geqslant \rho\left(\frac{\pi \alpha^{*}}{\sin \pi \rho} \cos \rho \theta+\int_{a a_{1}^{1 / \rho}}^{a a_{2}^{1 / \rho}} \frac{\left(\beta^{*} a^{-\rho}-\alpha^{*} t^{-\rho}\right)(t+b)}{t^{2}+2 b t+1} d t\right),
$$

или, вспоминая, что $b=\cos \theta$,

$$
\sigma_{\rho}(f) \geqslant \rho\left(\frac{\pi \alpha^{*}}{\sin \pi \rho} \cos \rho \theta+\int_{a a_{1}^{1 / \rho}}^{a a_{2}^{1 / \rho}} \frac{\left(\beta^{*} a^{-\rho}-\alpha^{*} t^{-\rho}\right)(t+\cos \theta)}{t^{2}+2 t \cos \theta+1} d t\right) .
$$

Наконец, используя свободу в выборе $a>0$, приходим к требуемой оценке (1.1):

$$
\sigma_{\rho}(f) \geqslant \rho\left(\frac{\pi \alpha^{*}}{\sin \pi \rho} \cos \rho \theta+\max _{a>0} \int_{a a_{1}^{1 / \rho}}^{a a_{2}^{1 / \rho}} \frac{\left(\beta^{*} a^{-\rho}-\alpha^{*} t^{-\rho}\right)(t+\cos \theta)}{t^{2}+2 t \cos \theta+1} d t\right) .
$$

Осталось рассмотреть случай измеримой последовательности нулей целой функции, когда существует предел

$$
\lim _{r \rightarrow+\infty} \frac{N(r)}{r^{\rho}}=\alpha^{*}=\beta^{*} .
$$

Здесь для любых $\varepsilon>0$ и $r>0$ найдется число $c$ такое, что при всех $\tau \geqslant c / r$ выполняется

$$
\Delta^{*}(r \tau) \geqslant \beta_{\varepsilon}^{*}=\frac{\beta^{*}}{1+\varepsilon} .
$$

Оценивая интеграл в (2.3), с учетом формулы (2.8) имеем

$$
\begin{aligned}
\int_{0}^{+\infty} & \Delta^{*}(r \tau) K(\tau) d \tau \geqslant \int_{c / r}^{+\infty} \Delta^{*}(r \tau) K(\tau) d \tau \geqslant \beta_{\varepsilon}^{*} \int_{c / r}^{+\infty} K(\tau) d \tau \\
= & \beta_{\varepsilon}^{*}\left(I_{1}-\int_{0}^{c / r} K(\tau) d \tau\right)=\beta_{\varepsilon}^{*} \frac{\pi \rho}{\sin \pi \rho} \cos \rho \theta+o(1), \quad r \rightarrow+\infty .
\end{aligned}
$$


Переход в (2.3) к верхнему пределу при $r \rightarrow+\infty$ дает

$$
\sigma_{\rho}(f) \geqslant \frac{\sigma_{\rho}(F)}{2} \geqslant \beta_{\varepsilon}^{*} \frac{\pi \rho}{\sin \pi \rho} \cos \rho \theta
$$

что при $\varepsilon \rightarrow 0$ приводит к оценке, совпадающей при $\alpha^{*}=\beta^{*}$ с неравенством (1.1). Действительно, когда $\alpha^{*}=\beta^{*}$, имеем $a_{1}=a_{2}=1$ и интеграл в оценке (1.1) пропадает.

\section{§ 3. Доказательство теоремы 1: построение экстремального примера}

Сейчас нам необходимо построить такую последовательность $\widetilde{\Lambda}=\left(\lambda_{n}\right)_{n=1}^{\infty}$, лежащую в угле $\Gamma_{\theta}=\{z \in \mathbb{C}:|\arg z| \leqslant \theta\}, \theta \in[0, \pi / 2]$, с усредненной считающей функцией $N_{\widetilde{\Lambda}}(r)$, чтобы выполнялись равенства

$$
\bar{\Delta}_{\rho}^{*}(\widetilde{\Lambda})=\varlimsup_{r \rightarrow+\infty} \frac{N_{\widetilde{\Lambda}}(r)}{r^{\rho}}=\beta^{*}, \quad \Delta_{\rho}^{*}(\widetilde{\Lambda})=\varliminf_{r \rightarrow+\infty} \frac{N_{\widetilde{\Lambda}}(r)}{r^{\rho}}=\alpha^{*},
$$

а $\rho$-тип экстремальной целой функции

$$
f_{0}(z)=\prod_{\lambda_{n} \in \widetilde{\Lambda}}\left(1-\frac{z}{\lambda_{n}}\right)
$$

составленной по $\widetilde{\Lambda}$, вычислялся согласно формуле

$$
\sigma_{\rho}\left(f_{0}\right)=\rho\left(\frac{\pi \alpha^{*}}{\sin \pi \rho} \cos \rho \theta+\max _{a>0} \int_{a a_{1}^{1 / \rho}}^{a a_{2}^{1 / \rho}} \frac{\left(\beta^{*} a^{-\rho}-\alpha^{*} t^{-\rho}\right)(t+\cos \theta)}{t^{2}+2 t \cos \theta+1} d t\right)
$$

где $a_{1}$ и $a_{2}-$ корни уравнения (1.2): $a \ln (e / a)=\alpha^{*} / \beta^{*}$.

Пусть сначала $\alpha^{*}=0$. В этом случае корнями уравнения (1.2) являются числа $a_{1}=0, a_{2}=e$, и оценка (1.1) принимает вид

$$
\begin{aligned}
\sigma_{\rho}(f) & \geqslant \rho \max _{a>0} \int_{0}^{a e^{1 / \rho}} \frac{\beta^{*} a^{-\rho}(\tau+\cos \theta)}{\tau^{2}+2 \tau \cos \theta+1} d \tau \\
& =\left.\frac{\beta^{*} \rho}{2} \max _{a>0} \frac{\ln \left(\tau^{2}+2 \tau \cos \theta+1\right)}{a^{\rho}}\right|_{0} ^{a e^{1 / \rho}}=\frac{\beta^{*} e \rho}{2} \max _{b>0} \frac{\ln \left(b^{2}+2 b \cos \theta+1\right)}{b^{\rho}} .
\end{aligned}
$$

На последнем шаге вычислений мы обозначили $b=a e^{1 / \rho}$. Этим доказана оценка (1.3). Точность этой оценки вытекает из следующего результата А. Ю. Попова (см. [9; теорема 3.1]), который сформулируем в удобной для нас форме: тип каждой целой функции $f(z)$ порядка $\rho \in(0,1)$ с нулями, расположенными в угле раствора $2 \theta \in[0, \pi]$ и имеющими верхнюю $\rho$-плотность $\bar{\Delta}_{\rho}=\beta$, удовлетворяет оценке

$$
\sigma_{\rho}(f) \geqslant \frac{\beta}{2} \max _{a>0} \frac{\ln \left(a^{2}+2 a \cos \theta+1\right)}{a^{\rho}} ;
$$

существует целая функция $f_{0}(z)$, реализующая равенство в этой оценке. 
Действительно, в силу неравенства (2.2) экстремальная функция, найденная А. Ю. Поповым, обеспечивает равенство в оценке теоремы 2 , поскольку с учетом этой оценки имеем

$$
\begin{aligned}
& \frac{\beta^{*} e \rho}{2} \max _{a>0} \frac{\ln \left(a^{2}+2 a \cos \theta+1\right)}{a^{\rho}} \leqslant \sigma_{\rho}\left(f_{0}\right)=\frac{\beta}{2} \max _{a>0} \frac{\ln \left(a^{2}+2 a \cos \theta+1\right)}{a^{\rho}} \\
& \leqslant \frac{\beta^{*} e \rho}{2} \max _{a>0} \frac{\ln \left(a^{2}+2 a \cos \theta+1\right)}{a^{\rho}} .
\end{aligned}
$$

$\mathrm{K}$ тому же нетрудно проверить, что нули функции $f_{0}(z)$ обладают нижними $\rho$-плотностями $\underline{\Delta}_{\rho}=\underline{\Delta}_{\rho}^{*}=0$ (см. также $\S 7$, замечание 2 об экстремальных функциях). Часть теоремы 1 , соответствующая случаю $\alpha^{*}=0$, доказана. Тем самым справедливость теоремы 2 установлена.

Рассмотрим теперь случай $0<\alpha^{*} \leqslant \beta^{*}$. Распределим искомую последовательность $\widetilde{\Lambda}$ одинаково на сторонах угла $\Gamma_{\theta}$, положив

$$
\left|\lambda_{2 k-1}\right|=\left|\lambda_{2 k}\right|=r_{k}, \quad \arg \lambda_{n}=(-1)^{n} \theta
$$

и образуем функцию

$$
f_{0}(z)=\prod_{n=1}^{\infty}\left(1-\frac{z}{r_{n} e^{i \theta}}\right)\left(1-\frac{z}{r_{n} e^{-i \theta}}\right)=\prod_{n=1}^{\infty}\left(1-\frac{2 z}{r_{n}} \cos \theta+\left(\frac{z}{r_{n}}\right)^{2}\right) .
$$

Используя такую форму записи экстремальной функции, сведем задачу к построению положительной последовательности

$$
|\widetilde{\Lambda}|:=\left(r_{n}\right)_{n=1}^{\infty}, \quad 0<r_{1}=\cdots=r_{n_{1}}<r_{n_{1}+1}=\cdots=r_{n_{2}}<r_{n_{2}+1}=\cdots
$$

с так подобранными кратностями $n_{1}, n_{2}, \ldots$, чтобы $|\widetilde{\Lambda}|$ обеспечивала минимальное значение для типа $\sigma_{\rho}\left(f_{0}\right)$ и удовлетворяла в соответствии с $(3.1)$ условиям

$$
\begin{aligned}
& B:=\varlimsup_{r \rightarrow+\infty} \frac{N(r)}{r^{\rho}}=\varlimsup_{r \rightarrow+\infty} \frac{N_{\widetilde{\Lambda}}(r)}{2 r^{\rho}}=\frac{\beta^{*}}{2}, \\
& A:=\varliminf_{r \rightarrow+\infty} \frac{N(r)}{r^{\rho}}=\varliminf_{r \rightarrow+\infty} \frac{N_{\widetilde{\Lambda}}(r)}{2 r^{\rho}}=\frac{\alpha^{*}}{2} .
\end{aligned}
$$

Здесь мы обозначили считающую и усредненную считающую функции последовательности $|\widetilde{\Lambda}|$ через $n(r)$ и $N(r)$ соответственно и воспользовались очевидными равенствами (в последовательность $\widetilde{\Lambda}$ члены входят сопряженными парами)

$$
n(r)=\frac{n_{\widetilde{\Lambda}}(r)}{2}, \quad N(r)=\frac{N_{\widetilde{\Lambda}}(r)}{2}, \quad r>0 .
$$

Непосредственно проверяемая формула

$$
r_{n}=\min \{r: n(r) \geqslant n\}, \quad n \in \mathbb{N},
$$

заменяет построение положительной последовательности (3.3) поиском ее считающей функции $n(r)$ или усредненной считающей функции $N(r)$. Нахождением этих функций мы сейчас и займемся. 
Для $r \in\left[r_{n_{k}}, r_{n_{k+1}}\right)$ имеем

$$
n(r)=n_{k}, \quad N(r)=N\left(r_{n_{k}}\right)+n_{k} \ln \frac{r}{r_{n_{k}}}, \quad k \in \mathbb{N} .
$$

Нам будет удобней перейти к функциям

$$
\omega(x):=n\left(e^{x}\right), \quad \Omega(x):=N\left(e^{x}\right) .
$$

График функции $\Omega(x)=\int_{0}^{x} \omega(t) d t$ представляет собой ломаную, $k$-е звено ко-
торой имеет уравнение

$$
\Omega(x)=\Omega\left(\ln r_{n_{k}}\right)+n_{k}\left(x-\ln r_{n_{k}}\right), \quad x \in\left[\ln r_{n_{k}}, \ln r_{n_{k+1}}\right) .
$$

Приступим к построению этого графика. Нам придется исследовать отдельно два случая: $0<\alpha^{*}=\beta^{*}$ и $0<\alpha^{*}<\beta^{*}$.

Пусть вначале $0<\alpha^{*}=\beta^{*}$ (т.е. $0<A=B$ ). Рассмотрим функцию $y_{A}(x)=A e^{\rho x}, x>0$, и проведем к графику $G_{A}$ этой функции касательные $l_{j}$ с угловыми коэффициентами, равными последовательным натуральным числам $j \in \mathbb{N}$. Нетрудно найти абсциссы $x_{j}$ точек касания: $x_{j}=(1 / \rho) \ln (j / \rho A)$, $j \in \mathbb{N}$. Пусть $y=\Omega_{1}(x)$ - уравнение ломаной, $j$-е звено которой является отрезком касательной $l_{j}$, содержащим точку касания $\left(x_{j}, A e^{\rho x_{j}}\right)$. В силу выпуклости функции $y_{A}(x)$ график этой ломаной расположен ниже графика $G_{A}$. Кроме того, по лемме $[13 ;$ гл. $3, \S 1]$ на каждом отрезке $\left[x_{j}, x_{j+1}\right]$ выполняется соотношение

$$
0 \leqslant y_{A}(x)-\Omega_{1}(x) \leqslant \frac{1}{4}\left(x_{j+1}-x_{j}\right)=\frac{1}{4 \rho} \ln \frac{j+1}{j}<\frac{1}{4 \rho j} .
$$

Таким образом,

$$
0 \leqslant y_{A}(x)-\Omega_{1}(x) \rightarrow 0, \quad x \rightarrow+\infty .
$$

Отсюда следует, что существует предел

$$
\lim _{r \rightarrow+\infty} \frac{N(r)}{r^{\rho}}=\lim _{r \rightarrow+\infty} \frac{\Omega_{1}(x)}{e^{\rho x}}=A=B,
$$

причем

$$
N(r) \leqslant A r^{\rho}, \quad r>0 .
$$

Теперь, используя (2.4), (3.2), запишем в прежних обозначениях представление

$$
\begin{aligned}
\sigma_{0}(r) & :=\ln \max _{|z|=r}\left|f_{0}(z)\right|=\ln \prod_{n=1}^{\infty}\left(1+\frac{2 r}{r_{n}} \cos \theta+\left(\frac{r}{r_{n}}\right)^{2}\right) \\
& =\sum_{n=1}^{\infty} \ln \left(1+\frac{2 r}{r_{n}} \cos \theta+\left(\frac{r}{r_{n}}\right)^{2}\right)=2 r^{\rho} \int_{0}^{+\infty} \Delta^{*}(r \tau) K(\tau) d \tau .
\end{aligned}
$$

Поскольку $\Delta^{*}(r)=N(r) / r^{\rho} \leqslant A$, то с учетом формулы (2.8) имеем

$$
\frac{\sigma_{0}(r)}{r^{\rho}} \leqslant 2 A I_{1}=2 A \int_{0}^{+\infty} K(\tau) d \tau=2 A \frac{\pi \rho}{\sin \pi \rho} \cos \rho \theta
$$


Переход к верхнему пределу при $r \rightarrow+\infty$ показывает, что $\rho$-тип построенной целой функции $f_{0}(z)$ удовлетворяет неравенству

$$
\sigma_{\rho}\left(f_{0}\right) \leqslant 2 A \frac{\pi \rho \cos \rho \theta}{\sin \pi \rho}=\alpha^{*} \frac{\pi \rho \cos \rho \theta}{\sin \pi \rho} .
$$

Обратное неравенство справедливо для $\rho$-типа каждой целой функции порядка $\rho \in(0,1)$ с измеримыми нулями усредненной $\rho$-плотности $\Delta^{*}=\alpha^{*}$, расположенными в угле раствора $2 \theta \leqslant \pi$, поскольку из (1.1) при $\alpha^{*}=\beta^{*}$ получаем

$$
\sigma_{\rho}(f) \geqslant \alpha^{*} \frac{\pi \rho \cos \rho \theta}{\sin \pi \rho} .
$$

В самом деле, в случае, когда $\alpha^{*}=\beta^{*}$, корнями уравнения (1.2) служат числа $a_{1}=a_{2}=1$ и интеграл в оценке (1.1) исчезает. Итак, теорема 1 при $\alpha^{*}=\beta^{*}$ доказана, что говорит о справедливости утверждения теоремы 3.

Продолжим построение экстремальной функции в ситуации, когда $\alpha^{*}<\beta^{*}$, т.е. $A<B$. В этом случае наряду с функциями $y_{A}(x)$ и $\Omega_{1}(x)$ рассмотрим функцию $y_{B}(x)=B e^{\rho x}, x>0$, и выберем какую-либо строго возрастающую последовательность натуральных чисел $m_{n}$, удовлетворяющую условию

$$
\frac{m_{n+1}}{m_{n}} \nearrow+\infty, \quad n \rightarrow \infty .
$$

Пусть $y=\Omega_{2}(x)$ - уравнение ломаной, снова составленное из уравнений последовательных отрезков касательных к графику $G_{B}$ функции $y_{B}(x)$ с угловыми коэффициентами, совпадающими с выбранными числами $m_{n}$ из (3.5). Как и прежде, легко найти абсциссы $\zeta_{n}$ точек касания этой ломаной с графиком $G_{B}$, именно, $\zeta_{n}=(1 / \rho) \ln \left(m_{n} / \rho B\right)$. Легко видеть, что точки пересечения последовательных звеньев ломаной лежат ниже графика $G_{A}$ в силу условия (3.5). Этого можно добиться уже начиная со второго звена за счет выбора углового коэффициента $m_{2}$ (достаточно взять $\left.m_{2}>\left(a_{2} / a_{1}\right) m_{1}\right)$. Определим теперь абсциссы $x_{n}^{(1)}$ и $x_{n}^{(2)}$ точек пересечения графика ломаной $y=\Omega_{2}(x)$ с графиком $G_{A}$. Для этого вычислим различными способами угловой коэффициент касательной к графику $G_{B}$, являющейся при условии $B>A$ также и секущей графика $G_{A}$. Имеем

$$
\frac{B e^{\rho \zeta_{n}}-A e^{\rho x_{n}^{(j)}}}{\zeta_{n}-x_{n}^{(j)}}=B \rho e^{\rho \zeta_{n}}, \quad j=1,2, \quad n \in \mathbb{N} .
$$

Разделим обе части этого равенства на $B e^{\rho \zeta_{n}} /\left(\zeta_{n}-x_{n}^{(j)}\right)$ и обозначим $y_{j}=$ $\zeta_{n}-x_{n}^{(j)}$. После преобразования полученного соотношения $1-(A / B) e^{-\rho y_{j}}=\rho y_{j}$, к виду $\left(1-\rho y_{j}\right) e^{\rho y_{j}}=A / B$ запишем

$$
e^{\rho y_{j}} \ln \frac{e}{e^{\rho y_{j}}}=\frac{A}{B}=\frac{\alpha^{*}}{\beta^{*}} .
$$

Отсюда получаем $e^{\rho y_{j}}=a_{2 / j}$, т.е. $y_{j}=\zeta_{n}-x_{n}^{(j)}=(1 / \rho) \ln a_{2 / j}, j=1,2$, где $a_{1}$, $a_{2}$ - корни уравнения (1.2). Таким образом,

$$
x_{n}^{(1)}=\zeta_{n}-\frac{1}{\rho} \ln a_{2}, \quad x_{n}^{(2)}=\zeta_{n}-\frac{1}{\rho} \ln a_{1}, \quad n \in \mathbb{N} .
$$


Выпуклость функций $y_{A}(x)$ и $y_{B}(x)$ приводит к соотношениям

$$
\Omega_{2}(x)=B e^{\rho \zeta_{n}}+\rho B e^{\rho \zeta_{n}}\left(x-\zeta_{n}\right) \in\left[A e^{\rho x}, B e^{\rho x}\right], \quad x \in\left[x_{n}^{(1)}, x_{n}^{(2)}\right], \quad n \in \mathbb{N} .
$$

Кроме того, по построению имеем

$$
\Omega_{2}\left(\zeta_{n}\right)=y_{B}\left(\zeta_{n}\right), \quad n \in \mathbb{N} .
$$

Введем функцию

$$
\Omega(x)=\max \left\{\Omega_{1}(x), \Omega_{2}(x)\right\}, \quad x>0 .
$$

Графиком функции (3.8) служит ломаная, абсциссы вершин которой (обозначим их $\ln r_{n}$ ) определяют некоторую последовательность. Для того чтобы усредненная считающая функция задавалась формулой $N\left(e^{x}\right)=\Omega(x)$, запишем каждый член последовательности с кратностью, равной разности угловых коэффициентов звеньев ломаной с рассматриваемой общей вершиной. Докажем, что полученная в результате новая последовательность $|\widetilde{\Lambda}|$ вида $(3.3)$ является искомой.

Действительно, в силу построения и с учетом (3.4), (3.6) имеем

$$
\begin{gathered}
\Omega(x) \leqslant y_{A}(x), \quad x \notin \bigcup_{n \in \mathbb{N}}\left[x_{n}^{(1)}, x_{n}^{(2)}\right], \\
y_{A}(x) \leqslant \Omega(x) \leqslant y_{B}(x), \quad x \in \bigcup_{n \in \mathbb{N}}\left[x_{n}^{(1)}, x_{n}^{(2)}\right] .
\end{gathered}
$$

Поскольку

$$
\lim _{n \rightarrow \infty} x_{n}^{(1)}=\lim _{n \rightarrow \infty}\left(\zeta_{n}-\frac{1}{\rho} \ln a_{2}\right)=\lim _{n \rightarrow \infty} \frac{1}{\rho} \ln \frac{m_{n}}{\rho B a_{2}}=+\infty,
$$

то из $(3.4),(3.7),(3.9),(3.10)$ заключаем, что выполнены предельные соотношения

$$
A=\varliminf_{x \rightarrow+\infty} \frac{\Omega(x)}{e^{\rho x}}=\varliminf_{r \rightarrow+\infty} \frac{N(r)}{r^{\rho}}, \quad B=\varlimsup_{x \rightarrow+\infty} \frac{\Omega(x)}{e^{\rho x}}=\varlimsup_{r \rightarrow+\infty} \frac{N(r)}{r^{\rho}} .
$$

Найденной последовательности $|\widetilde{\Lambda}|$, отвечает бесконечное произведение $f_{0}(z)$ вида (3.2). Для логарифма максимума модуля этой функции, как и в случае $A=B$, воспользуемся представлением

$$
\sigma_{0}(r)=2 r^{\rho} \int_{0}^{+\infty} \Delta^{*}(r \tau) K(\tau) d \tau, \quad \Delta^{*}(r)=\frac{N(r)}{r^{\rho}} .
$$

Вывод ключевого соотношения (1.1) основывался на оценке величины $\Delta^{*}(r)$ снизу. Для обоснования экстремального характера функции $f_{0}(z)$ потребуется оценить $\Delta^{*}(r)$ сверху. Вернемся к первоначальной переменной $x=\ln t$ и обозначим

$$
\xi_{n}=e^{\zeta_{n}}=\left(\frac{m_{n}}{\rho \beta^{*}}\right)^{1 / \rho}, \quad t_{n}^{(j)}=e^{x_{n}^{(j)}}=\xi_{n} a_{2 / j}^{-1 / \rho}, \quad j=1,2, \quad n \in \mathbb{N} .
$$


В силу (3.6), (3.9) имеем

$$
\begin{gathered}
N(t)=B \xi_{n}^{\rho}+\rho B \xi_{n}^{\rho} \ln \frac{t}{\zeta_{n}}, \quad t \in\left[t_{n}^{(1)}, t_{n}^{(2)}\right], \quad n \in \mathbb{N}, \\
N(t) \leqslant A t^{\rho}, \quad t \notin \bigcup_{n \in \mathbb{N}}\left[t_{n}^{(1)}, t_{n}^{(2)}\right] .
\end{gathered}
$$

Отсюда для величины $\Delta^{*}(r t):=N_{\Lambda}(r t) /(r t)^{\rho}$ при фиксированном $r>0$ извлекаем соотношения

$$
\begin{gathered}
\Delta^{*}(r t) \leqslant A, \quad t \notin \bigcup_{n \in \mathbb{N}}\left[\frac{t_{n}^{(1)}}{r}, \frac{t_{n}^{(2)}}{r}\right]=: T, \\
\Delta^{*}(r t)=\frac{B \xi_{n}^{\rho}}{(r t)^{\rho}}\left(1+\rho \ln \frac{r t}{\zeta_{n}}\right), \quad t \in\left[\frac{t_{n}^{(1)}}{r}, \frac{t_{n}^{(2)}}{r}\right], \quad n \in \mathbb{N} .
\end{gathered}
$$

Все нужные оценки усредненной считающей функции последовательности $\widetilde{\Lambda}$ получены.

Перейдем к обоснованию экстремальности целой функции (3.2), построенной по $\widetilde{\Lambda}$. Оценим сверху величину

$$
\begin{gathered}
\frac{\sigma_{0}(r)}{r^{\rho}}=\frac{\ln \max _{|z|=r}\left|f_{0}(z)\right|}{r^{\rho}}=2 \int_{0}^{+\infty} \Delta^{*}(r t) K(t) d t \\
K(t)=-t^{\rho}\left(\frac{t \cos \theta+1}{t^{2}+2 t \cos \theta+1}\right)^{\prime}
\end{gathered}
$$

Для этого, привлекая (3.12), (3.13), запишем

$$
\begin{aligned}
\int_{0}^{+\infty} & \Delta^{*}(r t) K(t) d t=\int_{\mathbb{R} \backslash T} \Delta^{*}(r t) K(t) d t+\int_{T} \Delta^{*}(r t) K(t) d t \\
\leqslant & A \int_{\mathbb{R} \backslash T} K(t) d t+\sum_{n=1}^{\infty} \int_{t_{n}^{(1)} / r}^{t_{n}^{(2)} / r} \frac{B \xi_{n}^{\rho}}{(r t)^{\rho}} \ln e\left(\frac{t r}{\xi_{n}}\right)^{\rho} K(t) d t \\
= & A \underbrace{\int_{0}^{+\infty} K(t) d t}_{I_{1}}+\sum_{n=1}^{\infty} \underbrace{\int_{t_{n}^{(1)} / r}^{t_{n}^{(2)} / r}\left(\frac{B \xi_{n}^{\rho}}{(r t)^{\rho}} \ln e\left(\frac{t r}{\xi_{n}}\right)^{\rho}-A\right) K(t) d t}_{S_{n}(r)} .
\end{aligned}
$$

Напомним (см. (2.8)), что $I_{1}=(\pi \rho / \sin \pi \rho) \cos \rho \theta$. Обозначим

$$
Q(t)=\frac{t \cos \theta+1}{t^{2}+2 t \cos \theta+1}
$$

и преобразуем слагаемые в сумме, интегрируя по частям каждое из них:

$$
\begin{aligned}
S_{n}(r) & :=-\int_{t_{n}^{(1)} / r}^{t_{n}^{(2)} / r}\left[\frac{B \xi_{n}^{\rho}}{r^{\rho}} \ln e\left(\frac{t r}{\xi_{n}}\right)^{\rho}-A t^{\rho}\right] Q^{\prime}(t) d t \\
& =-\left.Q(t)\left[\frac{B \xi_{n}^{\rho}}{r^{\rho}} \ln e\left(\frac{t r}{\xi_{n}}\right)^{\rho}-A t^{\rho}\right]\right|_{t_{n}^{(1)} / r} ^{t_{n}^{(2)} / r}+\rho \int_{t_{n}^{(1)} / r}^{t_{n}^{(2)} / r}\left(B\left(\frac{\xi_{n}}{r}\right)^{\rho}-A t^{\rho}\right) Q(t) \frac{d t}{t} .
\end{aligned}
$$


Подстановка обращается в нуль. В самом деле,

$$
\frac{B \xi_{n}^{\rho}}{r^{\rho}} \ln e\left(\frac{t_{n}^{(j)}}{\xi_{n}}\right)^{\rho}-A \frac{\left(t_{n}^{(j)}\right)^{\rho}}{r^{\rho}}=\frac{\left(t_{n}^{(j)}\right)^{\rho}}{r^{\rho}} B\left[a_{2 / j} \ln \frac{e}{a_{2 / j}}-\frac{A}{B}\right]=0,
$$

поскольку $a_{j}, j=1,2,-$ корни уравнения (1.2). Таким образом,

$$
S_{n}(r)=\rho \int_{t_{n}^{(1)} / r}^{t_{n}^{(2)} / r}\left(B\left(\frac{\xi_{n}}{r}\right)^{\rho}-A t^{\rho}\right) Q(t) \frac{d t}{t} .
$$

Теперь зафиксируем $j \in \mathbb{N}$ и оценим сумму

$$
S(r):=\sum_{n=1}^{\infty} S_{n}(r)=\sum_{n=1}^{j-1} S_{n}(r)+\sum_{n=j+2}^{\infty} S_{n}(r)+\left[S_{j}(r)+S_{j+1}(r)\right] .
$$

Будем использовать простые неравенства

$$
Q(t)=\frac{t \cos \theta+1}{t^{2}+2 t \cos \theta+1} \leqslant 1, \quad Q(t) \leqslant \frac{2}{t}, \quad t \in \mathbb{R}_{+} .
$$

Отсюда и из (3.14), пренебрегая отрицательными слагаемыми, получаем для величины $S_{n}(r)$ две оценки

$$
\begin{aligned}
S_{n}(r) & \leqslant \rho \int_{t_{n}^{(1)} / r}^{t_{n}^{(2)} / r} B\left(\frac{\xi_{n}}{r}\right)^{\rho} \frac{d t}{t}=B\left(\frac{\xi_{n}}{r}\right)^{\rho} \ln \frac{a_{2}}{a_{1}}, \\
S_{n}(r) & \leqslant 2 \rho \int_{t_{n}^{(1)} / r}^{t_{n}^{(2)} / r} B\left(\frac{\xi_{n}}{r}\right)^{\rho} \frac{d t}{t^{2}}=2 \rho B\left(\frac{\xi_{n}}{r}\right)^{\rho}\left(\frac{r}{t_{n}^{(1)}}-\frac{r}{t_{n}^{(2)}}\right) \\
& =2 \rho B\left(\frac{\xi_{n}}{r}\right)^{\rho-1}\left(a_{2}^{1 / \rho}-a_{1}^{1 / \rho}\right) .
\end{aligned}
$$

Для дальнейших оценок нам понадобится

ЛЕмма ШтольцА. Если положительные последовательности $a_{j} u b_{j}$, где $b_{j}$ строго монотонна, являются либо бесконечно большими, либо бесконечно малыми, то выполняется равенство

$$
\lim _{j \rightarrow \infty} \frac{a_{j}}{b_{j}}=\lim _{j \rightarrow \infty} \frac{a_{j+1}-a_{j}}{b_{j+1}-b_{j}}
$$

при условии, что предел справа существует.

Из (3.5) и (3.11) получаем условие

$$
\frac{\xi_{j+1}}{\xi_{j}}=\left(\frac{m_{j+1}}{m_{j}}\right)^{1 / \rho} \rightarrow \infty, \quad j \rightarrow \infty,
$$

которое влечет сходимость ряда $\sum_{j=1}^{\infty} \xi_{j}^{\rho-1}$. Применяя лемму Штольца, с учетом (3.18) находим

$$
\begin{gathered}
\lim _{j \rightarrow \infty} \frac{\sum_{n=1}^{j-1} \xi_{n}^{\rho}}{\xi_{j}^{\rho}}=\lim _{j \rightarrow \infty} \frac{\xi_{j}^{\rho}}{\xi_{j+1}^{\rho}-\xi_{j}^{\rho}}=\lim _{j \rightarrow \infty} \frac{1}{\left(\xi_{j+1} / \xi_{j}\right)^{\rho}-1}=0, \\
\lim _{j \rightarrow \infty} \frac{\sum_{n=j+2}^{\infty} \xi_{n}^{\rho-1}}{\xi_{j+1}^{\rho-1}}=\lim _{j \rightarrow \infty} \frac{-\xi_{j+2}^{\rho-1}}{\xi_{j+2}^{\rho-1}-\xi_{j+1}^{\rho-1}}=\lim _{j \rightarrow \infty} \frac{1}{\left(\zeta_{j+2} / \xi_{j+1}\right)^{1-\rho}-1}=0 .
\end{gathered}
$$


Теперь, используя (3.16), (3.17), показываем, что равномерно по $r \in\left[\xi_{j}, \xi_{j+1}\right]$ выполняются соотношения

$$
\begin{aligned}
\sum_{n=1}^{j-1} S_{n}(r) & \leqslant \sum_{n=1}^{j-1} B\left(\frac{\xi_{n}}{\xi_{j}}\right)^{\rho} \ln \frac{a_{2}}{a_{1}} \leqslant B \ln \frac{a_{2}}{a_{1}} \frac{\sum_{n=1}^{j-1} \xi_{n}^{\rho}}{\xi_{j}^{\rho}} \rightarrow 0, \quad j \rightarrow \infty, \\
\sum_{n=j+2}^{\infty} S_{n}(r) & \leqslant \sum_{n=j+2}^{\infty} 2 \rho B\left(\frac{\xi_{n}}{\xi_{j+1}}\right)^{\rho-1}\left(a_{2}^{1 / \rho}-a_{1}^{1 / \rho}\right) \\
& \leqslant 2 \rho B\left(a_{2}^{1 / \rho}-a_{1}^{1 / \rho}\right) \frac{\sum_{n=j+2}^{\infty} \xi_{n}^{\rho-1}}{\xi_{j+1}^{\rho-1}} \rightarrow 0, \quad j \rightarrow \infty .
\end{aligned}
$$

Для того чтобы оценить оставшуюся часть $S(r)$, в формуле $(3.14)$ для $S_{n}(r)$ сделаем замену переменной $t=1 / \tau$ :

$$
\begin{aligned}
S_{n}(r) & =\rho \int_{a_{2}^{-1 / \rho} \xi_{n} / r}^{a_{1}^{-1 / \rho} \xi_{n} / r}\left(B\left(\frac{\xi_{n}}{r}\right)^{\rho}-A t^{\rho}\right) \frac{t \cos \theta+1}{t^{2}+2 t \cos \theta+1} \frac{d t}{t} \\
& =\rho \int_{a_{1}^{1 / \rho} r / \xi_{n}}^{a_{2}^{1 / \rho} r / \xi_{n}}\left(B\left(\frac{r}{\xi_{n}}\right)^{-\rho}-A \tau^{-\rho}\right) \frac{\tau+\cos \theta}{\tau^{2}+2 \tau \cos \theta+1} d \tau .
\end{aligned}
$$

Таким образом, установлено равенство

$$
S_{n}(r)=\rho \varphi\left(\frac{r}{\xi_{n}}\right)
$$

в котором

$$
\varphi(a)=\int_{a a_{1}^{1 / \rho}}^{a a_{2}^{1 / \rho}} \frac{\left(B a^{-\rho}-A \tau^{-\rho}\right)(\tau+\cos \theta)}{\tau^{2}+2 \tau \cos \theta+1} d \tau, \quad a>0 .
$$

Изучим введенную функцию $\varphi(a)$. Покажем сначала, что

$$
\varphi(0+)=\varphi(+\infty)=0 .
$$

Интегрирование по частям дает для $\varphi(a)$ выражение

$$
\begin{aligned}
\varphi(a)= & \frac{1}{2} \int_{a a_{1}^{1 / \rho}}^{a a_{2}^{1 / \rho}}\left(B a^{-\rho}-A \tau^{-\rho}\right) d\left(\ln \left(\tau^{2}+2 \tau \cos \theta+1\right)\right) \\
= & \left.\frac{1}{2}\left[\left(B a^{-\rho}-A \tau^{-\rho}\right) \ln \left(\tau^{2}+2 \tau \cos \theta+1\right)\right]\right|_{a a_{1}^{1 / \rho}} ^{a a_{2}^{1 / \rho}} \\
& -\frac{1}{2} \int_{a a_{1}^{1 / \rho}}^{a a_{2}^{1 / \rho}} A \rho \tau^{-\rho-1} \ln \left(\tau^{2}+2 \tau \cos \theta+1\right) d \tau=: J_{1}(a)-J_{2}(a) .
\end{aligned}
$$

Проверим, что каждая из функций $J_{1}(a), J_{2}(a)$ удовлетворяет (3.22). Действительно, поскольку при $k=1,2$ имеем

$$
\begin{gathered}
\frac{\ln \left(a^{2} a_{k}^{2 / \rho}+2 a a_{k}^{1 / \rho} \cos \theta+1\right)}{a^{\rho}} \sim \frac{2 a a_{k}^{1 / \rho} \cos \theta}{a^{\rho}}=O\left(a^{1-\rho}\right) \rightarrow 0, \quad a \rightarrow 0+, \\
\frac{\ln \left(a^{2} a_{k}^{2 / \rho}+2 a a_{k}^{1 / \rho} \cos \theta+1\right)}{a^{\rho}} \sim \frac{2 \ln a}{a^{\rho}} \rightarrow 0, \quad a \rightarrow+\infty,
\end{gathered}
$$


то $J_{1}(0+)=J_{1}(+\infty)=0$. Далее,

$$
\begin{gathered}
\int_{a a_{1}^{1 / \rho}}^{a a_{2}^{1 / \rho}} \frac{\ln \left(\tau^{2}+2 \tau \cos \theta+1\right)}{\tau^{\rho+1}} d \tau \sim \int_{a a_{1}^{1 / \rho}}^{a a_{2}^{1 / \rho}} \frac{2 \cos \theta}{\tau^{\rho}} d \tau \\
=\frac{2 \cos \theta}{1-\rho}\left(a_{2}^{1 / \rho-1}-a_{1}^{1 / \rho-1}\right) a^{1-\rho} \rightarrow 0, \quad a \rightarrow 0+, \\
\int_{a a_{1}^{1 / \rho}}^{a a_{2}^{1 / \rho}} \frac{\ln \left(\tau^{2}+2 \tau \cos \theta+1\right)}{\tau^{\rho+1}} d \tau \sim \int_{a a_{1}^{1 / \rho}}^{a a_{2}^{1 / \rho}} \tau^{-\rho} \frac{2 \ln \tau}{\tau} d \tau \\
\leqslant a_{1}^{-1}\left(\frac{\ln ^{2} a a_{2}^{1 / \rho}}{a^{\rho}}-\frac{\ln ^{2} a a_{1}^{1 / \rho}}{a^{\rho}}\right) \rightarrow 0, \quad a \rightarrow+\infty .
\end{gathered}
$$

Значит, $J_{2}(0+)=J_{2}(+\infty)=0$, что приводит к равенствам

$$
\varphi(0+)=J_{1}(0+)-J_{2}(0+)=0, \quad \varphi(+\infty)=J_{1}(+\infty)-J_{2}(+\infty)=0 .
$$

Таким образом, (3.22) выполнено. Но теперь мы можем утверждать, что непрерывная на $\mathbb{R}_{+}$функция $\varphi(a)$ достигает своего максимума в некоторой точке $a_{0}$, т.е. $\max _{a>0} \varphi(a)=\varphi\left(a_{0}\right)>0$.

Используя равенство (3.21), оценим в сумме (3.15) последнее слагаемое

$$
J(r):=S_{j}(r)+S_{j+1}(r)=\rho\left[\varphi\left(\frac{r}{\xi_{j}}\right)+\varphi\left(\frac{r}{\xi_{j+1}}\right)\right] .
$$

Пусть сначала $r \in\left[\xi_{j}, \sqrt{\xi_{j} \xi_{j+1}}\right]$. Тогда $\varphi\left(r / \xi_{j}\right) \leqslant \varphi\left(a_{0}\right)$. Поскольку

$$
0<\frac{\xi_{j}}{\xi_{j+1}} \leqslant \frac{r}{\xi_{j+1}} \leqslant \sqrt{\frac{\xi_{j}}{\xi_{j+1}}} \rightarrow 0, \quad j \rightarrow \infty
$$

то благодаря (3.22) находим, что

$$
\max _{\xi_{j} \leqslant r \leqslant \sqrt{\xi_{j} \xi_{j+1}}} \varphi\left(\frac{r}{\xi_{j+1}}\right) \rightarrow 0, \quad j \rightarrow \infty .
$$

Пусть теперь $r \in\left[\sqrt{\xi_{j} \xi_{j+1}}, \xi_{j+1}\right]$. Тогда $\varphi\left(r / \xi_{j+1}\right) \leqslant \varphi\left(a_{0}\right)$. Так как

$$
\frac{r}{\xi_{j}} \geqslant \sqrt{\frac{\xi_{j+1}}{\xi_{j}}} \rightarrow \infty, \quad j \rightarrow \infty,
$$

то снова в силу (3.22) имеем

$$
\max _{\sqrt{\xi_{j} \xi_{j+1}} \leqslant r \leqslant \xi_{j+1}} \varphi\left(\frac{r}{\xi_{j}}\right) \rightarrow 0, \quad j \rightarrow \infty .
$$

В обоих случаях получаем

$$
J(r) \leqslant \rho \varphi\left(a_{0}\right)+o(1), \quad r \rightarrow+\infty .
$$


Сочетая эту оценку с (3.19), (3.20), запишем

$$
S(r)=\sum_{n=1}^{\infty} S_{n}(r)=\sum_{n=1}^{j-1} S_{n}(r)+\sum_{n=j+2}^{\infty} S_{n}(r)+J(r) \leqslant \rho \varphi\left(a_{0}\right)+o(1), \quad r \rightarrow+\infty .
$$

Возвращаясь к оценке типа построенной функции $f_{0}(z)$, имеем

$$
\begin{aligned}
\frac{\sigma_{0}(r)}{r^{\rho}} & =2 \int_{0}^{+\infty} \Delta^{*}(r t) K(t) d t \leqslant 2\left(A I_{1}+S(r)\right) \\
& \leqslant 2\left(A \frac{\pi \rho}{\sin \pi \rho} \cos \rho \theta+\rho \varphi\left(a_{0}\right)+o(1)\right), \quad r \rightarrow+\infty
\end{aligned}
$$

Переход к пределу при $r \rightarrow+\infty$ и сравнение с доказанным в 22 неравенством (1.1) приводит к требуемому результату:

$$
\begin{aligned}
\sigma_{\rho}\left(f_{0}\right) & =2 \rho\left(\frac{\pi A}{\sin \pi \rho} \cos \rho \theta+\varphi\left(a_{0}\right)\right) \\
& =2 \rho\left(\frac{\pi A}{\sin \pi \rho} \cos \rho \theta+\max _{a>0} \int_{a a_{1}^{1 / \rho}}^{a a_{2}^{1 / \rho}} \frac{\left(B a^{-\rho}-A t^{-\rho}\right)(t+\cos \theta)}{t^{2}+2 t \cos \theta+1} d t\right) \\
& =\rho\left(\frac{\pi \alpha^{*}}{\sin \pi \rho} \cos \rho \theta+\max _{a>0} \int_{a a_{1}^{1 / \rho}}^{a a_{2}^{1 / \rho}} \frac{\left(\beta^{*} a^{-\rho}-\alpha^{*} t^{-\rho}\right)(t+\cos \theta)}{t^{2}+2 t \cos \theta+1} d t\right) .
\end{aligned}
$$

Все случаи рассмотрены. Теорема 1 доказана.

В следующем параграфе мы несколько усиливаем эту теорему, показывая, что ее утверждение остается в силе для целых функций с нулями из более широкого класса множеств.

\section{§4. Обобщение теоремы 1}

Пусть $k^{*} \in[0,1]$ и $a_{1}, a_{2}\left(a_{1} \leqslant 1 \leqslant a_{2}\right)$ - корни уравнения $a \ln (e / a)=k^{*}$. Обозначим

$$
C_{\theta}^{*}\left(k^{*}, \rho\right):=\frac{\pi k^{*}}{\sin \pi \rho} \cos \rho \theta+\max _{a>0} \int_{a a_{1}^{1 / \rho}}^{a a_{2}^{1 / \rho}} \frac{\left(a^{-\rho}-k^{*} t^{-\rho}\right)(t+\cos \theta)}{t^{2}+2 t \cos \theta+1} d t,
$$

а в случае $\theta=0$ введем еще сокращенное обозначение

$$
C^{*}\left(k^{*}, \rho\right):=C_{0}^{*}\left(k^{*}, \rho\right)=\frac{\pi k^{*}}{\sin \pi \rho}+\max _{a>0} \int_{a a_{1}^{1 / \rho}}^{a a_{2}^{1 / \rho}} \frac{a^{-\rho}-k^{*} t^{-\rho}}{t+1} d t .
$$

Отметим, что при $\theta=\pi / 2$ имеем

$$
C_{\pi / 2}^{*}\left(k^{*}, \rho\right)=\frac{1}{2}\left(\frac{\pi k^{*}}{\sin (\pi \rho / 2)}+\max _{b>0} \int_{b a_{1}^{2 / \rho}}^{b a_{2}^{2 / \rho}} \frac{b^{-\rho / 2}-k^{*} t^{-\rho / 2}}{t+1} d t\right)=\frac{1}{2} C^{*}\left(k^{*}, \frac{\rho}{2}\right) .
$$


В самом деле, подставляя в (4.1) при $\theta=\pi / 2$ вместо параметра $a$ параметр $\sqrt{b}$ и делая замену переменной $\tau=t^{2}$, получаем

$$
\begin{aligned}
C_{\pi / 2}^{*}\left(k^{*}, \rho\right) & =\frac{\pi k^{*} \cos (\pi \rho / 2)}{\sin \pi \rho}+\max _{b>0} \int_{\sqrt{b} a_{1}^{1 / \rho}}^{\sqrt{b} a_{2}^{1 / \rho}} \frac{\left(b^{-\rho / 2}-k^{*} t^{-\rho}\right) t}{t^{2}+1} d t \\
& =\frac{1}{2}\left(\frac{\pi k^{*}}{\sin (\pi \rho / 2)}+\max _{b>0} \int_{b a_{1}^{2 / \rho}}^{b a_{2}^{2 / \rho}} \frac{b^{-\rho / 2}-k^{*} \tau^{-\rho / 2}}{\tau+1} d \tau\right)=\frac{1}{2} C^{*}\left(k^{*}, \frac{\rho}{2}\right) .
\end{aligned}
$$

Наконец, для $k^{*}=0$ обозначим

$$
C_{\theta}^{*}(\rho):=C_{\theta}^{*}(0, \rho)
$$

Прямое вычисление приводит к равенству

$$
C_{\theta}^{*}(0, \rho)=\max _{a>0} \int_{0}^{a e^{1 / \rho}} \frac{a^{-\rho}(t+\cos \theta)}{t^{2}+2 t \cos \theta+1} d t=\frac{e}{2} \max _{a>0} \frac{\ln \left(a^{2}+2 a \cos \theta+1\right)}{a^{\rho}},
$$

или

$$
C_{\theta}^{*}(0, \rho)=\frac{e}{2} C_{\theta}(\rho)
$$

где величина

$$
C_{\theta}(\rho)=\max _{a>0} \frac{\ln \left(a^{2}+2 a \cos \theta+1\right)}{a^{\rho}}
$$

введена в работе [9].

Для формулировки теоремы нам понадобится следующее определение.

Пусть $\theta \in[0, \pi / 2)$. Будем говорить, что последовательность $\Lambda=\left(\lambda_{n}\right)_{n=1}^{\infty}$ принадлежит классу $\widetilde{\Gamma}_{\theta}$, и писать $\Lambda \in \widetilde{\Gamma}_{\theta}$, если $\overline{\lim }_{n \rightarrow \infty}\left|\arg \lambda_{n}\right| \leqslant \theta$.

Будем писать $\Lambda \in \widetilde{\Gamma}_{\pi / 2}$, если все члены последовательности $\Lambda$, кроме, быть может, конечного числа, удовлетворяют условию $\left|\arg \lambda_{n}\right| \leqslant \pi / 2$.

Теорема 4. Пусть $\theta \in[0, \pi / 2]$. Тип каждой целой функции $f(z)$ порядка $\rho \in(0,1)$ с нулями $\Lambda_{f} \in \widetilde{\Gamma}_{\theta}$, имеюшими верхнюю и нижнюю усредненные $\rho$-плотности $\bar{\Delta}_{\rho}^{*}\left(\Lambda_{f}\right)=\beta^{*}, \Delta_{\rho}^{*}\left(\Lambda_{f}\right) \geqslant \alpha^{*}$, удовлетворяет точной оценке

$$
\sigma_{\rho}(f) \geqslant \rho \beta^{*} C_{\theta}^{*}\left(k^{*}, \rho\right), \quad k^{*}=\frac{\alpha^{*}}{\beta^{*}},
$$

где величина $C_{\theta}^{*}\left(k^{*}, \rho\right)$ определена в (4.1).

Равенство достигается на некоторой иелой функции с нулями $\widetilde{\Lambda}$, лежащими на лучах $\gamma_{ \pm \theta}:=\{z \in \mathbb{C}: \arg z= \pm \theta\}$, и имеющими усредненные $\rho$-плотности $\triangleq_{\rho}^{*}(\widetilde{\Lambda})=\alpha^{*}, \bar{\Delta}_{\rho}^{*}(\widetilde{\Lambda})=\beta^{*}$.

ДокАЗАТЕЛЬСтво. Если $\theta<\pi / 2$, то для произвольного $\theta^{\prime}>\theta$ найдется такое число $N$, что все нули функции $f(z)$ с номерами $n>N$ лежат в угле $\Gamma_{\theta^{\prime}}$. Такое же число найдется и в случае $\theta=\pi / 2$, когда нули попадают в угол $\Gamma_{\pi / 2}$. Во всех случаях функция $f(z)$ записывается в виде

$$
f(z)=\prod_{n<N}\left(1-\frac{z}{\lambda_{n}}\right) \prod_{n \geqslant N}\left(1-\frac{z}{\lambda_{n}}\right)=: \prod_{n<N}\left(1-\frac{z}{\lambda_{n}}\right) f_{N}(z),
$$


где первое произведение может и отсутствовать (произведение по пустому набору индексов считается равным единице). Функции $f(z)$ и $f_{N}(z)$ отличаются разве что полиномиальным множителем, поэтому их $\rho$-типы совпадают. Отличие в конечном наборе нулей не влияет на величины их усредненных $\rho$-плотностей, которые также совпадают:

$$
\bar{\Delta}_{\rho}^{*}\left(\Lambda_{f_{N}}\right)=\bar{\Delta}_{\rho}^{*}\left(\Lambda_{f}\right)=\beta^{*}, \quad \underline{\Delta}_{\rho}^{*}\left(\Lambda_{f_{N}}\right)=\underline{\Delta}_{\rho}^{*}\left(\Lambda_{f}\right) \geqslant \alpha^{*} .
$$

Но функция $f_{N}(z)$ удовлетворяет условиям теоремы 1 . Значит, для $f_{N}(z)$ справедливо полученное в процессе доказательства этой теоремы неравенство (2.10) (с $\theta^{\prime}$ вместо $\theta$ ), т.е.

$$
\sigma_{\rho}(f)=\sigma_{\rho}\left(f_{N}\right) \geqslant \rho\left(\frac{\pi \alpha^{*}}{\sin \pi \rho} \cos \rho \theta^{\prime}+\int_{a a_{1}^{1 / \rho}}^{a a_{2}^{1 / \rho}} \frac{\left(\beta^{*} a^{-\rho}-\alpha^{*} t^{-\rho}\right)\left(t+\cos \theta^{\prime}\right)}{t^{2}+2 t \cos \theta^{\prime}+1} d t\right) .
$$

Пользуясь сначала произвольностью $\theta^{\prime}>\theta$, а затем и свободой в выборе $a>0$, приходим опять к оценке (1.1), совпадающей с (4.3):

$$
\begin{aligned}
\sigma_{\rho}(f) & \geqslant \rho\left(\frac{\pi \alpha^{*}}{\sin \pi \rho} \cos \rho \theta+\max _{a>0} \int_{a a_{1}^{1 / \rho}}^{a a_{2}^{1 / \rho}} \frac{\left(\beta^{*} a^{-\rho}-\alpha^{*} t^{-\rho}\right)(t+\cos \theta)}{t^{2}+2 t \cos \theta+1} d t\right) \\
& =\rho \beta^{*}\left(\frac{\pi k^{*}}{\sin \pi \rho} \cos \rho \theta+\max _{a>0} \int_{a a_{1}^{1 / \rho}}^{a a_{2}^{1 / \rho}} \frac{\left(a^{-\rho}-k^{*} t^{-\rho}\right)(t+\cos \theta)}{t^{2}+2 t \cos \theta+1} d t\right) \\
& =\rho \beta^{*} C_{\theta}^{*}\left(k^{*}, \rho\right) .
\end{aligned}
$$

Последнее утверждение теоремы справедливо благодаря тому, что экстремальная последовательность нулей $\widetilde{\Lambda}=\left\{\lambda_{n}: \arg \lambda_{n}= \pm \theta\right\}$ принадлежит классу $\widetilde{\Gamma}_{\theta}$. Теорема 4 доказана.

Выделим два важных частных случая этой теоремы: $\theta=\pi / 2$ и $\theta=0$. При $\theta=\pi / 2$ корни $\Lambda_{f}=\left(\lambda_{n}\right)$ целых функций $f(z)$ лежат в замкнутой правой полуплоскости, т.е. $\operatorname{Re} \lambda_{n} \geqslant 0$. В этом случае справедливо такое утверждение.

СлеДСТвиЕ 1. Тип каждой целой функиии $f(z)$ порядка $\rho \in(0,1)$ с нулями $\Lambda_{f}$, лежащими в правой полуплоскости или на мнимой оси и имеющими верхнюю и нижнюю усредненные $\rho$-плотности $\bar{\Delta}_{\rho}^{*}\left(\Lambda_{f}\right)=\beta^{*}, \Delta_{\rho}^{*}\left(\Lambda_{f}\right) \geqslant \alpha^{*}$, удовлетворяет точной, достижимой оценке

$$
\sigma_{\rho}(f) \geqslant \frac{\rho \beta^{*}}{2}\left(\frac{\pi k^{*}}{\sin (\pi \rho / 2)}+\max _{b>0} \int_{b a_{1}^{2 / \rho}}^{b a_{2}^{2 / \rho}} \frac{b^{-\rho / 2}-k^{*} t^{-\rho / 2}}{t+1} d t\right)=\frac{\rho \beta^{*}}{2} C^{*}\left(k^{*}, \frac{\rho}{2}\right) .
$$

При $\theta=0$, как отмечалось во введении, из теоремы 4 следует обобщение теоремы 1 работы [7], доказанной для целых функций с положительными нулями.

СледствиЕ 2. Тип каждой целой функиии $f(z)$ порядка $\rho \in(0,1)$ с нулями $\Lambda_{f}=\left(\lambda_{n}\right)_{n=1}^{\infty}$, имеющими верхнюю и нижнюю усредненные $\rho$-плотности 
$\bar{\Delta}_{\rho}^{*}\left(\Lambda_{f}\right)=\beta^{*}, \Delta_{\rho}^{*}\left(\Lambda_{f}\right) \geqslant \alpha^{*}$ и такими, что $\arg \lambda_{n} \rightarrow 0, n \rightarrow \infty$, удовлетворяет точной, достижимой оценке

$\sigma_{\rho}(f) \geqslant \rho \beta^{*} C^{*}\left(k^{*}, \rho\right)=\rho \beta^{*}\left(\frac{\pi k^{*}}{\sin \pi \rho}+\max _{a>0} \int_{a a_{1}^{1 / \rho}}^{a a_{2}^{1 / \rho}} \frac{a^{-\rho}-k^{*} \tau^{-\rho}}{\tau+1} d \tau\right), \quad k^{*}=\frac{\alpha^{*}}{\beta^{*}}$, где $a_{1}, a_{2}\left(0 \leqslant a_{1} \leqslant 1 \leqslant a_{2} \leqslant e\right)-$ корни уравнения $a \ln (e / a)=\alpha^{*} / \beta^{*}$.

Отметим, что следствие 2 сохраняет точную оценку $\rho$-типа целых функций из [7; теорема 1], значительно расширяя множество комплексной плоскости, где могут располагаться все их нули. Например, при сохранении прочих условий этой теоремы оценка для $\rho$-типа остается точной и для целых функций с нулями, лежащими при любом $b>0$ в полуполосе $\{z=x+i y: x \geqslant 0,|y| \leqslant b\}$ или даже находящимися во множестве $\left\{z=x+i y: x \geqslant|y|^{\delta}\right\}$, где $\delta>1$. В $\S 5$ мы находим точную оценку типа целых функций порядка $\rho \in(0, m), m \in \mathbb{N}$, с нулями на еще более широких множествах.

ЗАмечание 1 . Пусть $\theta \in[0, \pi / 2)$. В условии теоремы 4 требуется, чтобы при любом $\theta^{\prime}>\theta$ вне угла $\Gamma_{\theta^{\prime}}$ лежало не более конечного числа корней целой функции. Это требование можно несколько ослабить, разрешая вне указанных углов находиться и бесконечному множеству корней, но с нулевой $\rho$-плотностью. Аналогичное допущение действует и при $\theta=\pi / 2$, когда бесконечно много корней с нулевой $\rho$-плотностью может располагаться вне полуплоскости $\{\operatorname{Re} z \geqslant 0\}$. Доказательство теоремы при этом практически не меняется.

\section{§ 5. Расположение нулей целых функций на "правильных" лучах или в углах}

Изучим ситуацию, когда нули целой функции лежат на $m$ лучах, исходящих из начала координат и разбивающих комплексную плоскость на равные части, или расположены в множествах, близких к непересекающимся углам, биссектрисами которых служат эти лучи. Нас интересует величина наименьшего типа целой функции с таким расположением нулей, выраженная через их усредненные $\rho$-плотности. Аналогичная задача для обычных $\rho$-плотностей нулей, лежащих на лучах, решена в работе [11]. Здесь мы используем некоторые идеи из этой работы. Пусть, как и прежде, $\Gamma_{\theta}=\{z \in \mathbb{C}:|\arg z| \leqslant \theta\}$. Для $m \in \mathbb{N}$ обозначим

$$
V_{\theta, m}=\bigcup_{k=0}^{m-1} \Gamma_{\theta, k, m}
$$

где

$$
\begin{aligned}
\Gamma_{\theta, k, m} & =\exp \left(i \frac{(2 k+1) \pi}{m}\right) \Gamma_{\theta} \\
& =\left\{z \in \mathbb{C}:\left|\arg z \exp \left(-i \frac{(2 k+1) \pi}{m}\right)\right| \leqslant \theta\right\}, \quad k=0,1, \ldots, m-1 .
\end{aligned}
$$


Например, множество $V_{0,1}=\Gamma_{0,0,1}$ есть отрицательный луч $\mathbb{R}_{-}$, а множество $V_{0,2}$ представляет собой мнимую ось комплексной плоскости. Вообще для заданного $m \geqslant 2$ множество $V_{0, m}=\bigcup_{k=0}^{m-1} \Gamma_{0, k, m}$ состоит из $m$ лучей, делящих плоскость на равные углы. При этом лучи

$$
\Gamma_{0, k, m}=\left\{z \in \mathbb{C}: z=r \exp \left(i \frac{(2 k+1) \pi}{m}\right), r>0\right\}, \quad k=0,1, \ldots, m-1,
$$

являются биссектрисами углов $\Gamma_{\theta, k, m}$.

Нам понадобятся следующие определения. Пусть $\theta \in[0, \pi / 2 m)$. Будем говорить, что последовательность $\Lambda=\left(\lambda_{n}\right)_{n=1}^{\infty}$ принадлежит классу $\widetilde{V}_{\theta, m}$, и писать $\Lambda \in \widetilde{V}_{\theta, m}$, если для любого $\theta^{\prime} \in(\theta, \pi / 2 m)$ все члены последовательности $\Lambda$, кроме, возможно, конечного числа, принадлежат множеству $V_{\theta^{\prime}, m}$. Для значения $\theta=\pi / 2 m$ пишем $\Lambda \in \widetilde{V}_{\pi / 2 m, m}$, если каждый член последовательности $\Lambda$, кроме, возможно, конечного числа, входит в $V_{\pi / 2 m, m}$. Например, последовательность, члены которой лежат в (наименьших) вертикальных углах, ограниченных двумя прямыми $l_{\theta}:=\left\{z \in \mathbb{C}: z=t e^{i \theta}, t \in \mathbb{R}\right\}$ и $l_{-\theta}:=\left\{z \in \mathbb{C}: z=t e^{-i \theta}, t \in \mathbb{R}\right\}$, где $\theta \in[0, \pi / 4]$, входит в класс $\widetilde{V}_{\theta, 2}$. Эти определения оставляем в силе, если исключительное множество образовано не конечным числом членов, а некоторой подпоследовательностью нулевой $\rho$-плотности, или, что эквивалентно, нулевой усредненной $\rho$-плотности.

Целью параграфа является доказательство следующего результата.

Теорема 5. Пусть $m \in \mathbb{N} u \theta \in[0, \pi / 2 m]$. Тип каждой целой функиии $f(z)$ порядка $\rho \in(0, m)$ с нулями $\Lambda_{f} \in \widetilde{V}_{\theta, m}$ заданных усредненных $\rho$-плотностей $\bar{\Delta}_{\rho}^{*}\left(\Lambda_{f}\right)=\beta^{*}, \Delta_{\rho}^{*}\left(\Lambda_{f}\right) \geqslant \alpha^{*}$ удовлетворяет точной оценке

$$
\sigma_{\rho}(f) \geqslant \frac{\rho \beta^{*}}{m} C_{m \theta}^{*}\left(k^{*}, \frac{\rho}{m}\right), \quad k^{*}=\frac{\alpha^{*}}{\beta^{*}},
$$

где величина $C_{\theta}^{*}\left(k^{*}, \rho\right)$ определена в (4.1).

Равенство достигается на некоторой целой бункции с нулями $\Lambda^{*}$, одинаково распределенными на граничных лучах множества $V_{\theta, m}$ и имеющими усредненные $\rho$-плотности $\triangleq_{\rho}^{*}\left(\Lambda^{*}\right)=\alpha^{*}, \bar{\Delta}_{\rho}^{*}\left(\Lambda^{*}\right)=\beta^{*}$.

В качестве следствия из этой теоремы при $\theta=0$ получаем такой результат.

Теорема 6. Пусть $m \in \mathbb{N} u f(z)$ - целая функиия порядка $\rho \in(0, m)$, нули которой $\Lambda_{f}$ имеют заданные усредненные $\rho$-плотности $\bar{\Delta}_{\rho}^{*}\left(\Lambda_{f}\right)=\beta^{*}$, $\underline{\Delta}_{\rho}^{*}\left(\Lambda_{f}\right) \geqslant \alpha^{*}$ и расположены на лучах, делящих плоскость на равные углы, или, более общим образом, $\Lambda_{f} \in \widetilde{V}_{0, m}$. Тогда $\rho$-тип $f(z)$ удовлетворяет точной оценке

$$
\sigma_{\rho}(f) \geqslant \frac{\rho}{m}\left(\frac{\pi \alpha^{*}}{\sin (\pi \rho / m)}+\max _{a>0} \int_{a a_{1}^{m / \rho}}^{a a_{2}^{m / \rho}} \frac{\beta^{*} a^{-\rho / m}-\alpha^{*} \tau^{-\rho / m}}{\tau+1} d \tau\right),
$$

где $a_{1}, a_{2}\left(0 \leqslant a_{1} \leqslant 1 \leqslant a_{2} \leqslant e\right)$ - корни уравнения $a \ln (e / a)=\alpha^{*} / \beta^{*}$.

Равенство достигается на некоторой целой функиии с нулями, лежащими на таких лучах и имеющими усредненные $\rho$-плотности $\triangleq_{\rho}^{*}\left(\Lambda^{*}\right)=\alpha^{*} / m$, $\bar{\Delta}_{\rho}^{*}\left(\Lambda^{*}\right)=\beta^{*} / m$ накаждом из них. 
ДокАЗАТЕльство. Пусть $m \in \mathbb{N}$, и $f(z)$ - целая функция порядка $\rho \in(0, m)$ с нулями $\Lambda_{f}=\Lambda \in \widetilde{V}_{\theta, m}$. Считаем, что $f(0)=1$. По уже цитированной теореме Адамара такую функцию можно представить в виде произведения

$$
f(z)=e^{q(z)} \prod_{n=1}^{\infty}\left(1-\frac{z}{\lambda_{n}}\right) e^{p_{n}(z)}, \quad z \in \mathbb{C},
$$

в котором

$$
p_{n}(z)=\frac{z}{\lambda_{n}}+\frac{z^{2}}{2 \lambda_{n}^{2}}+\cdots+\frac{z^{p}}{p \lambda_{n}^{p}},
$$

а число $p$ и степерь многочлена $q(z)$ не превосходят $\rho$. Кроме того, $q(0)=0$ в силу нашего соглашения $f(0)=1$. Обозначим через

$$
\varepsilon_{j}=\exp \left(i \frac{2 \pi j}{m}\right), \quad j=0,1, \ldots, m-1,
$$

корни степени $m$ из единицы и рассмотрим, следуя [11], функцию

$$
F(z)=\prod_{j=0}^{m-1} f\left(\varepsilon_{j} z\right), \quad z \in \mathbb{C} .
$$

Положим

$$
\lambda_{n}=r_{n} \exp \left(i \frac{\pi\left(2 k_{n}+1\right)}{m}+\theta_{n}\right)=\mu_{n} \exp \left(i \frac{\pi\left(2 k_{n}+1\right)}{m}\right), \quad n \in \mathbb{N},
$$

где $\mu_{n}=r_{n} e^{i \theta_{n}},\left|\theta_{n}\right| \leqslant \theta, r_{n} \nearrow+\infty$, а $k_{n} \in\{0,1, \ldots, m-1\}$. Учитывая, что для любого многочлена $Q(z)=\sum_{s=1}^{m-1} Q_{s} z^{s}$ без свободного члена выполняется равенство

$$
\sum_{j=0}^{m-1} Q\left(\varepsilon_{j} z\right)=\sum_{j=0}^{m-1} \sum_{s=1}^{m-1} Q_{s} z^{s} \varepsilon_{j}^{s}=\sum_{s=1}^{m-1} Q_{s} z^{s} \sum_{j=0}^{m-1} \varepsilon_{j}^{s} \equiv 0, \quad z \in \mathbb{C},
$$

так же, как в работе [11], устанавливаем, что функция $F(z)$ имеет вид

$$
F(z)=\prod_{n=1}^{\infty}\left(1+\left(\frac{z}{\mu_{n}}\right)^{m}\right), \quad z \in \mathbb{C} .
$$

Введем функцию

$$
F_{1}(z)=F(z) \overline{F(\bar{z})}=\prod_{n=1}^{\infty}\left(1+\left(\frac{z}{\mu_{n}}\right)^{m}\right)\left(1+\left(\frac{z}{\bar{\mu}_{n}}\right)^{m}\right), \quad z \in \mathbb{C}
$$

(черта означает комплексное сопряжение), и положим

$$
F_{1}\left(\exp \left(\frac{i \pi}{m}\right) z\right)=\prod_{n=1}^{\infty}\left(1-\left(\frac{z}{\mu_{n}}\right)^{m}\right)\left(1-\left(\frac{z}{\bar{\mu}_{n}}\right)^{m}\right)=: L\left(z^{m}\right), \quad z \in \mathbb{C}
$$


где

$$
L(z)=\prod_{n=1}^{\infty}\left(1-\frac{z}{r_{n}^{m} e^{i m \theta_{n}}}\right)\left(1-\frac{z}{r_{n}^{m} e^{-i m \theta_{n}}}\right), \quad z \in \mathbb{C} .
$$

Обозначив $t_{n}=r_{n}^{m}, \psi_{n}=m \theta_{n}$, можем записать

$$
L(z)=\prod_{n=1}^{\infty}\left(1-\frac{z}{t_{n} e^{i \psi_{n}}}\right)\left(1-\frac{z}{t_{n} e^{-i \psi_{n}}}\right), \quad z \in \mathbb{C} .
$$

Поскольку $\left|\psi_{n}\right|=\left|m \theta_{n}\right| \leqslant m \theta \leqslant \pi / 2$, то последовательность нулей $\Lambda_{L}$ бесконечного произведения $L(z)$ принадлежит классу $\widetilde{\Gamma}_{m \theta}$. Найдем усредненные $\rho$-плотности нулей введенных функций.

Как обычно, через $n_{T}(r)$ и $N_{T}(r)$ мы обозначаем считающую и усредненную считающую функции последовательности $T=\left(t_{n}\right)_{n=1}^{\infty}$ и, чтобы избежать громоздких обозначений, через $n_{L}(r)$ и $N_{L}(r)$ - такие же считающие функции последовательности нулей функции $L(z)$. Поскольку эти нули входят в произведение попарно сопряженными, то выполняется равенство $n_{L}(r)=2 n_{T}(r)$. Так как $t_{n}=r_{n}^{m}$, то $n_{T}\left(r^{m}\right)=n_{\Lambda}(r)$. Поэтому, подсчитывая $N_{L}(r)$ с помощью замены переменной $x=t^{m}$, получаем

$$
N_{L}\left(r^{m}\right)=\int_{0}^{r^{m}} \frac{n_{L}(x)}{x} d x=\int_{0}^{r^{m}} \frac{2 n_{T}(x)}{x} d x=2 m \int_{0}^{r} \frac{n_{\Lambda}(t)}{t} d t=2 m N_{\Lambda}(r),
$$

т.е. $N_{L}\left(r^{m}\right)=2 m N_{\Lambda}(r)$. Следовательно,

$$
\bar{\Delta}_{\rho / m}^{*}\left(\Lambda_{L}\right)=\varlimsup_{r \rightarrow+\infty} \frac{N_{L}\left(r^{m}\right)}{\left(r^{m}\right)^{\rho / m}}=2 m \varlimsup_{r \rightarrow+\infty} \frac{N_{\Lambda}(r)}{r^{\rho}}=2 m \bar{\Delta}_{\rho}^{*}(\Lambda)=2 m \beta^{*}
$$

и, аналогично,

$$
\Delta_{\rho / m}^{*}\left(\Lambda_{L}\right) \geqslant 2 m \alpha^{*} .
$$

Легко сравнить $\rho$-типы целых функций $F_{1}(z)$ и $f(z)$. Действительно,

$$
\begin{aligned}
\max _{|z|=r}\left|F_{1}(z)\right| & =\max _{|z|=r}|F(z) \overline{F(\bar{z})}|=\max _{|z|=r}\left|\prod_{j=0}^{m-1} f\left(\varepsilon_{j} z\right) \overline{f\left(\overline{\varepsilon_{j} z}\right)}\right| \\
& \leqslant \prod_{j=0}^{m-1} \max _{|z|=r}\left|f\left(\varepsilon_{j} z\right)\right| \max _{|z|=r}\left|\overline{f\left(\overline{\varepsilon_{j} z}\right)}\right|=\left(\max _{|z|=r}|f(z)|\right)^{2 m} .
\end{aligned}
$$

Отсюда получаем неравенство $\sigma_{\rho}\left(F_{1}\right) \leqslant 2 m \sigma_{\rho}(f)$, или

$$
\sigma_{\rho}(f) \geqslant \frac{1}{2 m} \sigma_{\rho}\left(F_{1}\right) .
$$

Сравним теперь $\rho$-типы целых функций $F_{1}(z)$ и $L(z)$, опираясь на тождество $F_{1}(\exp (i \pi / m) z)=L\left(z^{m}\right)$. Имеем

$$
\begin{aligned}
\sigma_{\rho}\left(F_{1}\right) & =\varlimsup_{r \rightarrow+\infty} \frac{\ln \max _{|z|=r}\left|F_{1}(\exp (i \pi / m) z)\right|}{r^{\rho}} \\
& =\varlimsup_{r \rightarrow+\infty} \frac{\ln \max _{|z|=r}\left|L\left(z^{m}\right)\right|}{\left(r^{m}\right)^{\rho / m}}=\sigma_{\rho / m}(L) .
\end{aligned}
$$

Таким образом, $\sigma_{\rho / m}(L)=\sigma_{\rho}\left(F_{1}\right)$. 
Применяя к функции $L(z)$ оценку (4.3) с учетом соотношений

$$
\frac{\rho}{m} \in(0,1), \quad \bar{\Delta}_{\rho / m}^{*}\left(\Lambda_{L}\right)=2 m \beta^{*}, \quad \triangleq_{\rho / m}^{*}\left(\Lambda_{L}\right) \geqslant 2 m \alpha^{*},
$$

приходим к неравенству

$$
\sigma_{\rho / m}(L) \geqslant \frac{\rho}{m} 2 m \beta^{*} C_{m \theta}^{*}\left(k^{*}, \frac{\rho}{m}\right)=2 \rho \beta^{*} C_{m \theta}^{*}\left(k^{*}, \frac{\rho}{m}\right), \quad k^{*}=\frac{2 m \alpha^{*}}{2 m \beta^{*}}=\frac{\alpha^{*}}{\beta^{*}} .
$$

Используя это неравенство и установленную выше в (5.4) связь типов функций $f(z)$ и $F_{1}(z)$, заключаем, что для любой функции $f(z)$ порядка $\rho \in(0, m)$ с нулями $\Lambda_{f} \in \widetilde{V}_{\theta, m}$ усредненных $\rho$-плотностей $\bar{\Delta}_{\rho}^{*}(\Lambda)=\beta^{*}, \triangleq_{\rho}^{*}(\Lambda) \geqslant \alpha^{*}$ справедлива оценка

$$
\sigma_{\rho}(f) \geqslant \frac{1}{2 m} \sigma_{\rho}\left(F_{1}\right)=\frac{1}{2 m} \sigma_{\rho / m}(L) \geqslant \frac{1}{2 m} 2 \rho \beta^{*} C_{m \theta}^{*}\left(k^{*}, \frac{\rho}{m}\right)=\frac{\rho \beta^{*}}{m} C_{m \theta}^{*}\left(k^{*}, \frac{\rho}{m}\right)
$$

с постоянной $k^{*}=\alpha^{*} / \beta^{*}$. Значит, для всех целых функций из рассматриваемого класса выполняется неравенство (5.1).

Зафиксируем произвольно числа

$$
m \in \mathbb{N}, \quad \rho \in(0, m), \quad \theta \in\left[0, \frac{\pi}{2 m}\right], \quad \beta^{*}>0, \quad \alpha^{*} \in\left[0, \beta^{*}\right] .
$$

Для доказательства точности оценки (5.1) воспользуемся примером экстремальной целой функции

$$
f_{0}(z)=\prod_{n=1}^{\infty}\left(1-\frac{z}{s_{n} e^{i m \theta}}\right)\left(1-\frac{z}{s_{n} e^{-i m \theta}}\right)
$$

построенной в $\S 3$ для порядка $\rho / m \in(0,1)$. Последовательность

$$
\Lambda_{f_{0}}=\widetilde{\Lambda}=\left(s_{n} e^{ \pm i m \theta}\right)_{n=1}^{\infty}
$$

нулей этой функции обладает усредненными $(\rho / m)$-плотностями

$$
\Delta_{\rho / m}^{*}(\widetilde{\Lambda})=\alpha^{*}, \quad \bar{\Delta}_{\rho / m}^{*}(\widetilde{\Lambda})=\beta^{*},
$$

и расположена на лучах $\gamma_{ \pm m \theta}=\{z \in \mathbb{C}: \arg z= \pm m \theta\}$. Функция $f_{0}(z)$ доставляет равенство в (4.3), именно

$$
\sigma_{\rho / m}\left(f_{0}\right)=\frac{\rho}{m} \beta^{*} C_{m \theta}^{*}\left(k^{*}, \frac{\rho}{m}\right), \quad k^{*}=\frac{\alpha^{*}}{\beta^{*}} .
$$

Введем функцию $f^{*}(z):=f_{0}\left(-z^{m}\right)$. Ее нули образуют множество

$$
\Lambda_{f^{*}}=\widetilde{\Lambda}=\bigcup_{k=0}^{m-1}\left\{\lambda_{n, k}^{ \pm}\right\}_{n=1}^{\infty},
$$

где

$$
\lambda_{n, k}^{ \pm}=\sqrt[m]{s_{n}} \exp ( \pm i \theta) \exp \left(i \frac{\pi(2 k+1)}{m}\right), \quad n \in \mathbb{N}, \quad k=0,1, \ldots, m-1,
$$


расположенное на $2 m$ лучах

$$
\left\{z \in \mathbb{C}: z=t \exp \left(i\left( \pm \theta+\frac{\pi(2 k+1)}{m}\right)\right), t>0\right\}, \quad k=0,1, \ldots, m-1 .
$$

Поэтому $\widetilde{\Lambda} \in \widetilde{V}_{\theta, m}$. Кроме того, поскольку $n_{\Lambda^{*}}(r)=m n_{\widetilde{\Lambda}}\left(r^{m}\right)$, то

$$
N_{\Lambda^{*}}(r)=\int_{0}^{r} \frac{n_{\Lambda^{*}}(x)}{x} d x=m \int_{0}^{r} \frac{n_{\widetilde{\Lambda}}\left(x^{m}\right)}{x} d x=\int_{0}^{r^{m}} \frac{n_{\widetilde{\Lambda}}(t)}{t} d t=N_{\widetilde{\Lambda}}\left(r^{m}\right) .
$$

Это, в свою очередь, означает, что $\bar{\Delta}_{\rho}^{*}\left(\Lambda^{*}\right)=\beta^{*}, \Delta_{\rho}^{*}\left(\Lambda^{*}\right)=\alpha^{*}$. Действительно, например,

$$
\bar{\Delta}_{\rho}^{*}\left(\Lambda^{*}\right)=\varlimsup_{r \rightarrow+\infty} \frac{N_{\Lambda^{*}}(r)}{r^{\rho}}=\varlimsup_{r \rightarrow+\infty} \frac{N_{\widetilde{\Lambda}}\left(r^{m}\right)}{\left(r^{m}\right)^{\rho / m}}=\bar{\Delta}_{\rho / m}^{*}(\widetilde{\Lambda})=\beta^{*} .
$$

Как и ранее, из определения $f^{*}(z)=f_{0}\left(-z^{m}\right)$ следует, что $\sigma_{\rho}\left(f^{*}\right)=\sigma_{\rho / m}\left(f_{0}\right)$, а сопоставление с (5.5) приводит к заключению

$$
\sigma_{\rho}\left(f^{*}\right)=\sigma_{\rho / m}\left(f_{0}\right)=\frac{\rho}{m} \beta^{*} C_{m \theta}^{*}\left(k^{*}, \frac{\rho}{m}\right), \quad k^{*}=\frac{\alpha^{*}}{\beta^{*}} .
$$

Таким образом, $f^{*}(z)$ - требуемая экстремальная функция, реализующая равенство в (5.1). Теорема 5 доказана.

Если в этой теореме ограничение на нижнюю усредненную $\rho$-плотность отсутствует, т.е. $\alpha^{*}=0$, то получается следующее утверждение.

Теорема 7. Тип каждой челой функиии $f(z)$ порядка $\rho \in(0, m)$ с нулями $\Lambda_{f} \in \widetilde{V}_{\theta, m}$ усредненной верхней $\rho$-плотности $\bar{\Delta}_{\rho}^{*}\left(\Lambda_{f}\right)=\beta^{*}$ удовлетворяет точной, достижимой оценке

$$
\sigma_{\rho}(f) \geqslant \frac{\beta^{*} e \rho}{m} \max _{a>0} \frac{\ln \left(a^{2 m}+2 a^{m} \cos \theta+1\right)}{a^{\rho}} .
$$

В самом деле, достаточно в оценку (5.1) подставить величину (4.2) и заменить параметр $a$ на $a^{m}$.

В случае $m=1$ эта теорема несколько усиливает теорему 2 в части ослабления условий на расположение нулей целой функции.

Случай $m=2$ теоремы 5 и некоторые следствия из нее мы обсудим в следующем параграфе.

\section{§ 6. Расположение нулей целых функций на одной прямой или между двумя прямыми}

Выделим наиболее интересные для приложений случаи расположения нулей целых функций на одной прямой или между двумя параллельными (пересекающимися) прямыми, соответствующие значению $m=2$ теоремы 5 . Нам удобнее здесь рассматривать целые функции, имеющие последовательности нулей, 
“привязанные" не к мнимой, а к вещественной оси. Ясно, что соответствующий поворот комплексной плоскости не меняет ни величины типа целой функции, ни значений усредненных плотностей ее нулей. Сформулируем результат, вытекающий из теоремы 5 при $m=2$, в виде отдельного утверждения.

Теорема 8. Пусть $\theta \in[0, \pi / 4]$. Тип каждой целой функиии $f(z)$ поряд$\kappa a \rho \in(0,2)$ с нулями $\Lambda_{f} \in \widetilde{V}_{2, \theta}$ усредненных $\rho$-плотностей $\bar{\Delta}_{\rho}^{*}\left(\Lambda_{f}\right)=\beta^{*}$, $\Delta_{\rho}^{*}\left(\Lambda_{f}\right) \geqslant \alpha^{*}$, удовлетворяет точной оценке

$$
\begin{aligned}
\sigma_{\rho}(f) & \geqslant \frac{\rho \beta^{*}}{2} C_{2 \theta}\left(k^{*}, \frac{\rho}{2}\right) \\
& =\frac{\rho \beta^{*}}{2}\left(\frac{\pi k^{*}}{\sin (\pi \rho / 2)} \cos 2 \rho \theta+\max _{a>0} \int_{a a_{1}^{2 / \rho}}^{a a_{2}^{2 / \rho}} \frac{\left(a^{-\rho / 2}-k^{*} t^{-\rho / 2}\right)(t+\cos 2 \theta)}{t^{2}+2 t \cos 2 \theta+1} d t\right),
\end{aligned}
$$

где $k^{*}=\alpha^{*} / \beta^{*}$ и $a_{1}, a_{2}\left(0 \leqslant a_{1} \leqslant 1 \leqslant a_{2} \leqslant e\right)$ - корни уравнения $a \ln (e / a)=k^{*}$. Равенство достигается на некоторой целой функиии с нулями $\Lambda^{*}$, лежащими на прямых $l_{ \pm \theta}=\left\{z \in \mathbb{C}: z=t e^{ \pm i \theta}, t \in \mathbb{R}\right\}$ и имеющими усредненные $\rho$-плотности $\triangleq_{\rho}^{*}\left(\Lambda^{*}\right)=\alpha^{*}, \bar{\Delta}_{\rho}^{*}\left(\Lambda^{*}\right)=\beta^{*}$.

Для $\theta=0$ отсюда получаем

СлеДСтвие 3. Тип каждой целой функиии $f(z)$ порядка $\rho \in(0,2)$ с нулями

$$
\Lambda_{f}=\left\{\lambda_{n}\right\}: \frac{\operatorname{Im} \lambda_{n}}{\left|\lambda_{n}\right|} \rightarrow 0, \quad n \rightarrow \infty
$$

имеющими усредненные $\rho$-плотности $\bar{\Delta}_{\rho}^{*}\left(\Lambda_{f}\right)=\beta^{*}, \triangleq_{\rho}^{*}\left(\Lambda_{f}\right) \geqslant \alpha^{*}$, удовлетворяет точной, достижимой оченке

$$
\sigma_{\rho}(f) \geqslant \frac{\rho \beta^{*}}{2} C^{*}\left(k^{*}, \frac{\rho}{2}\right)=\frac{\rho \beta^{*}}{2}\left(\frac{\pi k^{*}}{\sin (\pi \rho / 2)}+\max _{a>0} \int_{a a_{1}^{2 / \rho}}^{a a_{2}^{2 / \rho}} \frac{a^{-\rho / 2}-k^{*} \tau^{-\rho / 2}}{\tau+1} d \tau\right),
$$

где $k^{*}=\alpha^{*} / \beta^{*}$ и $a_{1}, a_{2}\left(0 \leqslant a_{1} \leqslant 1 \leqslant a_{2} \leqslant e\right)-$ корни уравнения $a \ln (e / a)=k^{*}$.

Обратим внимание на то, что этот результат справедлив, в частности, в случаях, когда нули целой функции расположены:

a) на вещественной прямой;

b) между параллельными прямыми $\{z \in \mathbb{C}: \operatorname{Im} z= \pm h\}$ при любом $h>0$;

c) более общим образом во множестве

$$
\left\{z=x+i y \in \mathbb{C}:|x| \delta_{1}(x) \leqslant y \leqslant|x| \delta_{2}(x)\right\},
$$

где $\delta_{1}(x) \leqslant \delta_{2}(x), x \in \mathbb{R}$, и для $k=1,2$ функции $\delta_{k}(x) \rightarrow 0$ при $x \rightarrow \pm \infty$.

Интересно также отметить, что оценки величины $\rho$-типа из следствий 1 и 3 совпадают. Но следствие 1 справедливо для целых функций порядка $\rho \in(0,1)$ с нулями на оси или в полуплоскости, а следствие 3 - для целых функций порядка $\rho \in(0,2)$ с нулями на оси или "близкими" к ней. 
Особый интерес представляет поведение на мнимой оси целой функции с вещественными нулями, характеризуемое величинами $\varlimsup_{x \rightarrow \pm \infty} \ln \left(|f(i x)| /|x|^{\rho}\right)$, т.е. значениями в точках $\varphi= \pm \pi / 2$ индикатора

$$
h_{\rho}(f, \varphi):=\varlimsup_{r \rightarrow+\infty} \frac{\ln \left|f\left(r e^{i \varphi}\right)\right|}{r^{\rho}}, \quad-\pi \leqslant \varphi \leqslant \pi .
$$

В случае измеримой последовательности нулей, учитывая, что пределы в определениях ее плотностей и усредненных плотностей существуют одновременно, из [14; теорема 7.3.1], [15; теорема 8.2.1] можно извлечь следующий факт.

Пусть $f(z)$ - целая функция порядка $\rho \in(0,1)$, все нули которой $\Lambda_{f}=\Lambda$ являются вещественными. Тогда следующие два утверждения эквивалентны:

$$
\exists \lim _{r \rightarrow+\infty} \frac{N_{\Lambda}(r)}{r^{\rho}}=\beta^{*}, \quad \exists \lim _{x \rightarrow \pm \infty} \frac{\ln |f(i x)|}{|x|^{\rho}}=\frac{\pi \rho \beta^{*}}{2 \sin (\pi \rho / 2)} .
$$

Результат верен также и для канонических произведений порядка $\rho=1$.

В менее изученном случае целых функций с неизмеримыми вещественными нулями теорема 8 дает точную нижнюю оценку для значений $h_{\rho}(f, \pm \pi / 2)$.

Действительно, если $c \in \mathbb{R}, \lambda_{n} \in \mathbb{R}$ и

$$
f(z)=e^{c z} \prod_{n=1}^{\infty}\left(1-\frac{z}{\lambda_{n}}\right) e^{z / \lambda_{n}}, \quad F(z)=f(z) f(-z)=\prod_{n=1}^{\infty}\left(1-\left(\frac{z}{\lambda_{n}}\right)^{2}\right),
$$

то при вещественных $r>0$ нетрудно установить равенство (см. [11])

$$
\max _{|z|=r}|F(z)|=|F( \pm i r)|=\prod_{n=1}^{\infty}\left(1+\left(\frac{r}{\lambda_{n}}\right)^{2}\right)=|f( \pm i r)|^{2} .
$$

Эти же соотношения справедливы и для более простого произведения

$$
f(z)=\prod_{n=1}^{\infty}\left(1-\frac{z}{\lambda_{n}}\right), \quad \lambda_{n} \in \mathbb{R} .
$$

В любом случае имеем $\ln |f( \pm i r)=(1 / 2) \ln | F( \pm i r) \mid$, и это дает равенство

$$
h_{\rho}\left(f, \pm \frac{\pi}{2}\right)=\frac{1}{2} h_{\rho}\left(F, \pm \frac{\pi}{2}\right)=\frac{1}{2} \sigma_{\rho}(F) .
$$

Кроме того, очевидно, что

$$
\bar{\Delta}_{\rho}^{*}\left(\Lambda_{F}\right)=2 \bar{\Delta}_{\rho}^{*}\left(\Lambda_{f}\right), \quad \triangleq_{\rho}^{*}\left(\Lambda_{F}\right)=2 \underline{\Delta}_{\rho}^{*}\left(\Lambda_{f}\right) .
$$

На основании теоремы 8 заключаем, в частности, что индикатор в точках $\varphi= \pm \pi / 2$ любой целой функции нецелого порядка $\rho \in(0,2)$ с вещественными нулями заданных усредненных $\rho$-плотностей не меньше, чем минимальный возможный $\rho$-тип на классе таких функций. Дадим точную формулировку. 
Tеорема 9. Пусть $\rho \in(0,2), \rho \neq 1, \beta^{*}>0, \alpha^{*} \in\left[0, \beta^{*}\right], u f(z)-u е-$ лая функиия порядка $\rho$ с вещественными нулями $\rho$-плотностей $\bar{\Delta}_{\rho}^{*}\left(\Lambda_{f}\right)=\beta^{*}$, $\Delta_{\rho}^{*}\left(\Lambda_{f}\right) \geqslant \alpha^{*}$. Тогда

$$
h_{\rho}\left(f,-\frac{\pi}{2}\right)=h_{\rho}\left(f, \frac{\pi}{2}\right)=\varlimsup_{r \rightarrow+\infty} \frac{\ln |f( \pm i r)|}{r^{\rho}} \geqslant \frac{\rho \beta^{*}}{2} C^{*}\left(k^{*}, \frac{\rho}{2}\right), \quad k^{*}=\frac{\alpha^{*}}{\beta^{*}} .
$$

При $\rho=1$ результат верен для функииц вида

$$
f(z)=e^{c z} \prod_{n=1}^{\infty}\left(1-\frac{z}{\lambda_{n}}\right) \exp \left(\frac{z}{\lambda_{n}}\right), \quad \lambda_{n} \in \mathbb{R}, \quad c \in \mathbb{R},
$$

а именно, справедливо неравенство

$$
h\left(f, \pm \frac{\pi}{2}\right) \geqslant \frac{\beta}{2} C^{*}\left(k^{*}, \frac{1}{2}\right)=\frac{\pi \alpha^{*}}{2}+\max _{a>0} \Phi_{\alpha^{*}, \beta^{*}}(a),
$$

где величина

$$
\Phi_{\alpha^{*}, \beta^{*}}^{*}(a)=\frac{\beta^{*}}{2 \sqrt{a}} \ln \frac{1+a a_{2}^{2}}{1+a a_{1}^{2}}-\alpha^{*} \operatorname{arctg} \frac{\left(a_{2}-a_{1}\right) \sqrt{a}}{1+a a_{1} a_{2}}
$$

получена прямым подсчетом интеграла в (4.1) при $\rho=1 / 2$.

Можно ли дать оценки индикатора целой функции в точках, отличных от $\varphi= \pm \pi / 2$ ? Как показывает следующий результат, в некоторых случаях разработанный метод предоставляет возможность получать точные оценки индикатора не только в отдельных точках, но и для значений аргумента из определенных промежутков. Пусть $f(z)$ - целая функция порядка $\rho \in(0,1)$ с отрицательными нулями $\lambda_{n}=-r_{n}, 0<r_{n} \nearrow+\infty$. Тогда справедливо представление

$$
f(z)=\prod_{n=1}^{\infty}\left(1+\frac{z}{r_{n}}\right), \quad z \in \mathbb{C}
$$

причем

$$
\begin{aligned}
\left|f\left(r e^{i \theta}\right)\right|^{2} & =f\left(r e^{i \theta}\right) \overline{f\left(r e^{i \theta}\right)} \\
& =\prod_{n=1}^{\infty}\left(1+\frac{r e^{i \theta}}{r_{n}}\right)\left(1+\frac{r e^{-i \theta}}{r_{n}}\right)=\prod_{n=1}^{\infty}\left(1+2 \frac{r}{r_{n}} \cos \theta+\left(\frac{r}{r_{n}}\right)^{2}\right) .
\end{aligned}
$$

Но в правой части равенства

$$
2 \ln \left|f\left(r e^{i \theta}\right)\right|=\sum_{n=1}^{\infty} \ln \left(1+\frac{2 r}{r_{n}} \cos \theta+\left(\frac{r}{r_{n}}\right)^{2}\right)
$$

без труда узнаем величину $\sigma(r)$, на оценке которой основывалось доказательство теоремы 1. Следует учесть только, что, в отличие от ситуации в этой теореме, считающая функция нулей уменьшается в два раза, поскольку нули теперь располагаются не на двух лучах - сторонах угла, а на одной отрицательной полуоси. Отметим также, что выбор последовательности модулей нулей экстремальной целой функции из теоремы 1 не зависел от раствора угла. Указанные обстоятельства позволяют сформулировать следующие утверждения. 
Теорема 10. Пусть $\beta^{*}>0, \alpha^{*} \in\left[0, \beta^{*}\right]$ u $f(z)$ - целая функиия порядка $\rho \in$ $(0,1)$ с отрицательными нулями $\Lambda_{f}$ усредненных $\rho$-плотностей $\bar{\Delta}_{\rho}^{*}\left(\Lambda_{f}\right)=\beta^{*}$, $\underline{\Delta}_{\rho}^{*}\left(\Lambda_{f}\right) \geqslant \alpha^{*}$. Тогда для каждого значения $\theta \in[-\pi / 2, \pi / 2]$ справедлива точная оценка

$$
h_{\rho}(f, \theta)=\varlimsup_{r \rightarrow+\infty} \frac{\ln \left|f\left(r e^{i \theta}\right)\right|}{r^{\rho}} \geqslant \rho \beta^{*} C_{\theta}^{*}\left(k^{*}, \rho\right), \quad k^{*}=\frac{\alpha^{*}}{\beta^{*}},
$$

или, в развернутом виде,

$$
h_{\rho}(f, \theta) \geqslant \rho\left(\frac{\pi \alpha^{*}}{\sin \pi \rho} \cos \rho \theta+\max _{a>0} \int_{a a_{1}^{1 / \rho}}^{a a_{2}^{1 / \rho}} \frac{\left(\beta^{*} a^{-\rho}-\alpha^{*} t^{-\rho}\right)(t+\cos \theta)}{t^{2}+2 t \cos \theta+1} d t\right),
$$

где $a_{1}, a_{2}\left(0 \leqslant a_{1} \leqslant 1 \leqslant a_{2} \leqslant e\right)$ - корни уравнения $a \ln (e / a)=\alpha^{*} / \beta^{*}$.

Существует целая функция, обладающая отрицательными нулями усредненных $\rho$-плотностей $\bar{\Delta}_{\rho}^{*}\left(\Lambda_{f}\right)=\beta^{*}, \Delta_{\rho}^{*}\left(\Lambda_{f}\right)=\alpha^{*}$, индикатор которой при всех $\theta \in[-\pi / 2, \pi / 2]$ обеспечивает равенство в (6.2).

При $\alpha^{*}=0$ отсюда следует такое утверждение.

Теорема 11. Индикатор каждой целой функции $f(z)$ порядка $\rho \in(0,1)$ с отрицательными нулями $\Lambda_{f}$ усредненной верхней $\rho$-плотности $\bar{\Delta}_{\rho}^{*}\left(\Lambda_{f}\right)=\beta^{*}$ для каждого значения $\theta \in[-\pi / 2, \pi / 2]$ удовлетворяет точному неравенству

$$
h_{\rho}(f, \theta) \geqslant \frac{\beta^{*} e \rho}{2} \max _{a>0} \frac{\ln \left(a^{2}+2 a \cos \theta+1\right)}{a^{\rho}} .
$$

Существует челая функиия, обладающая отрищательными нулями усредненной $\rho$-плотности $\bar{\Delta}_{\rho}^{*}\left(\Lambda_{f}\right)=\beta^{*}$, индикатор которой при всех $\theta \in[-\pi / 2, \pi / 2]$ обеспечивает равенство в (6.3).

При $\alpha^{*}=\beta^{*}$ из (6.2) вытекает неравенство

$$
h_{\rho}(f, \theta) \geqslant \rho \frac{\pi \beta^{*}}{\sin \pi \rho} \cos \rho \theta, \quad \theta \in\left[-\frac{\pi}{2}, \frac{\pi}{2}\right] .
$$

Однако для целых функций с измеримой последовательностью отрицательных нулей известна асимптотика $f(z)$ при $z \rightarrow \infty$, откуда точно находится величина индикатора $h_{\rho}(f, \theta)$ при всех $\theta$ (см., например, [14; гл. 7]):

$$
h_{\rho}(f, \theta)=\rho \frac{\pi \beta^{*}}{\sin \pi \rho} \cos \rho \theta, \quad \theta \in[-\pi, \pi] .
$$

\section{§ 7. Свойства экстремальной величины $C_{\theta}^{*}\left(k^{*}, \rho\right)$}

Наименьший возможный $\rho$-тип целых функций с нулями на рассмотренных в предыдущих параграфах множествах выражается через величину (4.1):

$$
C_{\theta}^{*}\left(k^{*}, \rho\right):=\frac{\pi k^{*}}{\sin \pi \rho} \cos \rho \theta+\max _{a>0} \int_{a a_{1}^{1 / \rho}}^{a a_{2}^{1 / \rho}} \frac{\left(a^{-\rho}-k^{*} t^{-\rho}\right)(t+\cos \theta)}{t^{2}+2 t \cos \theta+1} d t,
$$


имеющую весьма сложный вид. Приведем здесь некоторые ее свойства, полезные на практике, что, в частности, позволит нам несколько полнее охарактеризовать экстремальные целые функции. Выведем также простые оценки для величины $C_{\theta}^{*}\left(k^{*}, \rho\right)$ через элементарные функции и неэлементарную функцию (см.(4.2))

$$
C_{\theta}(\rho)=\max _{a>0} \frac{\ln \left(a^{2}+2 a \cos \theta+1\right)}{a^{\rho}},
$$

исследованную ранее в [9; теорема 3.2].

Приступая к изучению функции $C_{\theta}^{*}\left(k^{*}, \rho\right)$, покажем сначала, что она является возрастающей и выпуклой по переменной $k^{*}$ на отрезке $[0,1]$ при каждом фиксированном значении параметров $\rho \in(0,1)$ и $\theta \in[0, \pi / 2]$. Поскольку максимум выпуклых и возрастающих функций также является функцией выпуклой и возрастающей, то достаточно показать, что при любых фиксированных $\rho \in(0,1), \theta \in[0, \pi / 2]$ и $a>0$ этими же свойствами обладает функция

$$
\varphi(x)=\varphi_{\rho, \theta, a}(x):=\frac{\pi x}{\sin \pi \rho} \cos \rho \theta+\int_{a a_{1}^{1 / \rho}}^{a a_{2}^{1 / \rho}} \frac{\left(a^{-\rho}-x t^{-\rho}\right)(t+\cos \theta)}{t^{2}+2 t \cos \theta+1} d t,
$$

в определении которой $a_{1}=a_{1}(x)$ и $a_{2}=a_{2}(x)$ суть корни уравнения

$$
a \ln \frac{e}{a}=x, \quad x \in[0,1], \quad 0 \leqslant a_{1} \leqslant 1 \leqslant a_{2} \leqslant e .
$$

Из этого уравнения легко получаем, что

$$
a_{j}^{\prime}(x)=-\frac{1}{\ln a_{j}(x)}, \quad 1-\frac{x}{a_{j}(x)}=\ln a_{j}(x), \quad j=1,2 .
$$

С учетом записанных равенств найдем производную функции $\varphi(x)$, полагая ради краткости $\cos \theta=b$ и $R(t)=(t+b) /\left(t^{2}+2 t b+1\right)$. Имеем

$$
\begin{aligned}
\varphi^{\prime}(x)= & \frac{\pi}{\sin \pi \rho} \cos \rho \theta+\left(a^{-\rho}-x a^{-\rho} a_{2}^{-1}\right) R\left(a a_{2}^{1 / \rho}\right) \frac{a}{\rho} a_{2}^{1 / \rho-1} a_{2}^{\prime} \\
& \quad-\left(a^{-\rho}-x a^{-\rho} a_{1}^{-1}\right) R\left(a a_{1}^{1 / \rho}\right) \frac{a}{\rho} a_{1}^{1 / \rho-1} a_{1}^{\prime}-\int_{a a_{1}^{1 / \rho}}^{a a_{2}^{1 / \rho}} t^{-\rho} R(t) d t \\
= & \frac{\pi}{\sin \pi \rho} \cos \rho \theta+\frac{1}{\rho}\left[\left(a^{1-\rho} a_{1}^{1 / \rho-1}\right) R\left(a a_{1}^{1 / \rho}\right)-\left(a^{1-\rho} a_{2}^{1 / \rho-1}\right) R\left(a a_{2}^{1 / \rho}\right)\right] \\
& \quad-\int_{a a_{1}^{1 / \rho}}^{a a_{2}^{1 / \rho}} t^{-\rho} R(t) d t .
\end{aligned}
$$

Обозначив для сокращения записи $t_{j}=a a_{j}^{1 / \rho}, j=1,2$, получим

$$
\begin{aligned}
\varphi^{\prime}(x) & =\frac{\pi}{\sin \pi \rho} \cos \rho \theta-\frac{1}{\rho}\left(t_{2}^{1-\rho} R\left(t_{2}\right)-t_{1}^{1-\rho} R\left(t_{1}\right)\right)-\int_{t_{1}}^{t_{2}} t^{-\rho} R(t) d t \\
& =\frac{\pi}{\sin \pi \rho} \cos \rho \theta-\frac{1}{\rho} \int_{t_{1}}^{t_{2}} t^{-\rho}(t R(t))^{\prime} d t .
\end{aligned}
$$


Последнее равенство легко проверяется:

$$
\begin{gathered}
\left(\frac{x^{1-\rho} R(x)}{\rho}+\int_{0}^{x} t^{-\rho} R(t) d t\right)^{\prime}=\frac{(1-\rho) x^{-\rho} R(x)+x^{1-\rho} R^{\prime}(x)}{\rho}+x^{-\rho} R(x) \\
=\frac{1}{\rho}\left(x^{-\rho} R(x)+x^{1-\rho} R^{\prime}(x)\right)=\frac{x^{-\rho}}{\rho}\left(R(x)+x R^{\prime}(x)\right)=\frac{x^{-\rho}}{\rho}(x R(x))^{\prime} .
\end{gathered}
$$

Таким образом, мы пришли к формуле

$$
\varphi^{\prime}(x)=\frac{\pi}{\sin \pi \rho} \cos \rho \theta-\frac{1}{\rho} \int_{a a_{1}^{1 / \rho}}^{a a_{2}^{1 / \rho}} t^{-\rho}(t R(t))^{\prime} d t
$$

причем подынтегральное выражение положительно:

$$
(t R(t))^{\prime}=\left(\frac{t^{2}+t b}{t^{2}+2 t b+1}\right)^{\prime}=\frac{b t^{2}+2 t+b}{\left(t^{2}+2 t b+1\right)^{2}}>0, \quad t \geqslant 0 .
$$

Из приведенных выше соотношений $a_{j}^{\prime}(x)=-1 / \ln a_{i}(x), j=1,2$, следует, что функция $a_{1}(x)$ возрастает на отрезке $[0,1]$, а функция $a_{2}(x)$ убывает на этом же отрезке. Значит, интеграл в формуле (7.1) берется по стягивающемуся с ростом $x \in[0,1]$ отрезку. Величина интеграла при этом убывает, что влечет возрастание $\varphi^{\prime}(x)$. Следовательно, функция $\varphi(x)$ выпукла на $[0,1]$. Кроме того, при всех $x \in[0,1]$ выполнено

$$
\varphi^{\prime}(x) \geqslant \varphi^{\prime}(0)=\frac{\pi}{\sin \pi \rho} \cos \rho \theta-\frac{1}{\rho} \int_{0}^{a e^{1 / \rho}} t^{-\rho}(t R(t))^{\prime} d t=\frac{1}{\rho} \int_{a e^{1 / \rho}}^{+\infty} t^{-\rho}(t R(t))^{\prime} d t>0 .
$$

Поэтому функция $\varphi(x)$ также возрастает на $[0,1]$. При вычислении несобственного интеграла мы воспользовались результатом из [12; гл. 2, раздел 2.2.9].

Поскольку

$$
\begin{gathered}
\varphi(1)=\pi \frac{\cos \rho \theta}{\sin \pi \rho}, \\
\varphi(0)=\int_{0}^{a e^{1 / \rho}} a^{-\rho} R(t) d t=\frac{e}{2} \frac{\ln \left(1+2 a e^{1 / \rho} \cos \theta+\left(a e^{1 / \rho}\right)^{2}\right)}{\left(a e^{1 / \rho}\right)^{\rho}} \leqslant \frac{e}{2} C_{\theta}(\rho),
\end{gathered}
$$

то в силу выпуклости $\varphi(x)$ имеем

$$
\varphi_{\rho, \theta, a}(x)=\varphi(x) \leqslant \varphi(1) x+\varphi(0)(1-x) \leqslant \frac{\pi x \cos \rho \theta}{\sin \pi \rho}+\frac{e}{2} C_{\theta}(\rho)(1-x) .
$$

Тогда

$$
C_{\theta}^{*}\left(k^{*}, \rho\right)=\max _{a>0}\left\{\varphi_{\rho, \theta, a}\left(k^{*}\right)\right\} \leqslant \frac{\pi k^{*} \cos \rho \theta}{\sin \pi \rho}+\frac{e}{2} C_{\theta}(\rho)\left(1-k^{*}\right) .
$$

Снова используя выпуклость $\varphi(x)$ и учитывая, что $\varphi^{\prime}(1)=\pi \cos \rho \theta / \sin \pi \rho$, запишем

$$
\varphi_{\rho, \theta, a}(x)=\varphi(x) \geqslant \varphi(1)+\varphi^{\prime}(1)(x-1)=\frac{\pi \cos \rho \theta}{\sin \pi \rho}+\frac{\pi \cos \rho \theta}{\sin \pi \rho}(x-1)=\frac{\pi x \cos \rho \theta}{\sin \pi \rho}
$$


при всех $x \in[0,1]$. Отсюда заключаем, что

$$
C_{\theta}^{*}\left(k^{*}, \rho\right)=\max _{a>0}\left\{\varphi_{\rho, \theta, a}\left(k^{*}\right)\right\} \geqslant \frac{\pi k^{*} \cos \rho \theta}{\sin \pi \rho} .
$$

Аналогичным образом получаем следующую оценку:

$$
\begin{gathered}
\varphi_{\rho, \theta, a}(x)=\varphi(x) \geqslant \varphi(0)+\varphi^{\prime}(0) x=\frac{e}{2} \frac{\ln \left(1+2 a e^{1 / \rho} \cos \theta+\left(a e^{1 / \rho}\right)^{2}\right)}{\left(a e^{1 / \rho}\right)^{\rho}} \\
+x\left(\frac{\pi \cos \rho \theta}{\sin \pi \rho}-\frac{1}{\rho} \int_{0}^{a e^{1 / \rho}} t^{-\rho}(t R(t))^{\prime} d t\right) .
\end{gathered}
$$

Если точка $a_{0}=a_{0}(\rho, \theta)$ такова, что

$$
C_{\theta}(\rho)=\max _{a>0} \frac{\ln \left(1+2 a \cos \theta+a^{2}\right)}{a^{\rho}}=\frac{\ln \left(1+2 a_{0} \cos \theta+a_{0}^{2}\right)}{a_{0}^{\rho}},
$$

TO

$$
\begin{aligned}
C_{\theta}^{*}\left(k^{*}, \rho\right) & =\max _{a>0}\left\{\varphi_{\rho, \theta, a}\left(k^{*}\right)\right\} \geqslant \varphi_{\rho, \theta, a_{0} e^{-1 / \rho}}\left(k^{*}\right) \\
& =\frac{e}{2} C_{\theta}(\rho)+k^{*}\left(\frac{\pi \cos \rho \theta}{\sin \pi \rho}-\frac{1}{\rho} \int_{0}^{a_{0}} t^{-\rho}(t R(t))^{\prime} d t\right) .
\end{aligned}
$$

Полученную информацию соберем в отдельное утверждение.

ПрЕДЛОЖЕНИЕ 1. При любых $k^{*} \in[0,1], \rho \in(0,1), \theta \in[-\pi / 2, \pi / 2]$ справедливы следующие оченки:

$$
\begin{gathered}
\frac{\pi k^{*}}{\sin \pi \rho} \cos \rho \theta \leqslant C_{\theta}^{*}\left(k^{*}, \rho\right) \leqslant \frac{\pi k^{*}}{\sin \pi \rho} \cos \rho \theta+\frac{e}{2} C_{\theta}(\rho)\left(1-k^{*}\right), \\
C_{\theta}^{*}\left(k^{*}, \rho\right) \geqslant \frac{\pi k^{*}}{\sin \pi \rho} \cos \rho \theta+\frac{e}{2} C_{\theta}(\rho)-\frac{k^{*}}{\rho} B_{\theta}(\rho),
\end{gathered}
$$

¿де

$$
B_{\theta}(\rho)=\int_{0}^{a_{0}} t^{-\rho} \frac{t^{2} \cos \theta+2 t+\cos \theta}{\left(t^{2}+2 t \cos \theta+1\right)^{2}} d t
$$

и $a_{0}-$ точка, на которой достигается максимум в определении $C_{\theta}(\rho)$.

Равенства достигаются по крайней мере в крайних точках $k^{*}=0$ и $k^{*}=1$, поскольку из приведенных оценок вытекают соотношения

$$
\begin{gathered}
C_{\theta}^{*}(0, \rho)=\frac{e}{2} C_{\theta}(\rho)=\frac{e}{2} \max _{a>0} \frac{\ln \left(a^{2}+2 a \cos \theta+1\right)}{a^{\rho}}, \\
C_{\theta}^{*}(1, \rho)=\frac{\pi}{\sin \pi \rho} \cos \rho \theta .
\end{gathered}
$$

ЗАмечАниЕ 2. Доказанное при фиксированных

$$
\rho \in(0,1), \quad a>0, \quad \theta \in\left[-\frac{\pi}{2}, \frac{\pi}{2}\right]
$$


строгое возрастание функции $\varphi(x)=\varphi_{\rho, \theta, a}(x)$ влечет строгое возрастание по переменной $k^{*}$ максимума

$$
C_{\theta}^{*}\left(k^{*}, \rho\right)=\max _{a>0}\left\{\varphi_{\rho, \theta, a}\left(k^{*}\right)\right\} .
$$

Этот факт позволяет установить интересное свойство экстремальных функций, доставляющих равенства в оценках из теорем 1-9. Именно, каждая такая функция имеет минимально допустимую условиями своих теорем нижнюю усредненную $\rho$-плотность нулей. В самом деле, если, например, в теореме 4 нули $\Lambda_{f}$ функции $f(z)$ имеют нижнюю усредненную $\rho$-плотность

$$
\triangleq_{\rho}^{*}\left(\Lambda_{f}\right)=\alpha_{1}^{*}>\alpha^{*}
$$

то величина ее $\rho$-типа подчинена условию

$$
\sigma_{\rho}(f) \geqslant \rho \beta^{*} C_{\theta}^{*}\left(\frac{\alpha_{1}^{*}}{\beta^{*}}, \rho\right)>\rho \beta^{*} C_{\theta}^{*}\left(\frac{\alpha^{*}}{\beta^{*}}, \rho\right) .
$$

Такая функция $f(z)$ не может быть экстремальной в теореме 4. Это же замечание справедливо и в отношении других теорем.

\section{Список литературы}

[1] Б. Я. Левин, Распределение корней целых функиий, Гостехиздат, М., 1956, 632 с.; англ. пер.: В. Ja. Levin, Distribution of zeros of entire functions, Amer. Math. Soc., Providence, RI, 1964, viii+493 pp.

[2] А. Ю. Попов, "О полноте в пространствах аналитических функций систем экспонент с вещественными показателями заданной верхней плотности”, Вестн. Моск. ун-та. Сер. 1 Матем. Мех., 1999, №5, 48-52; англ. пер.: А. Yu. Popov, "Completeness of exponential systems with real exponents of a prescribed under density in spaces of analytic functions", Mosc. Univ. Math. Bull., 54:5 (1999), 47-51.

[3] R. M. Redheffer, "On even entire functions with zeros having a density", Trans. Amer. Math. Soc., 77 (1954), 32-61.

[4] А. Ю. Попов, "Наименьший возможный тип при порядке $\rho<1$ канонических произведений с положительными нулями заданной верхней $\rho$-плотности”, Вестн. Моск. ун-та. Сер. 1 Матем. Мех., 2005, № 1, 31-36; англ. пер.: A. Yu. Popov, "The least possible type under the order $\rho<1$ of canonical products with positive zeros of a given upper $\rho$-density", Mosc. Univ. Math. Bull., 60:1 (2005), 32-36.

[5] Г. Г. Брайчев, В. Б. Шерстюков, "О наименьшем возможном типе целых функций порядка $\rho \in(0,1)$ с положительными нулями”, Изв. РАН. Сер. матем., 75:1 (2011), 3-28; англ. пер.: G. G. Braichev, V. B. Sherstyukov, "On the least possible type of entire functions of order $\rho \in(0,1)$ with positive zeros", Izv. Math., 75:1 (2011), 1-27.

[6] Г. Г. Брайчев, В. Б. Шерстюков, "О росте целых функций с дискретно измеримыми нулями", Матем. заметки, 91:5 (2012), 674-690; англ. пер.: G. G. Braichev, V.B. Sherstyukov, "On the growth of entire functions with discretely measurable zeros", Math. Notes, 91:5 (2012), 630-644.

[7] Г. Г. Брайчев, "Наименьший тип целой функции порядка $\rho \in(0,1)$ с положительными корнями заданных усредненных плотностей”, Матем. сб., 203:7 (2012), 31-56; англ. пер.: G. G. Braichev, "The least type of an entire function of order $\rho \in(0,1)$ having positive zeros with prescribed averaged densities", Sb. Math., 203:7 (2012), 950-975. 
[8] Г. Г. Брайчев, "Точные оценки типа целой функции порядка меньше единицы с нулями на луче заданных усредненных плотностей”, Докл. PAH, 445:6 (2012), 615-617; англ. пер.: G. G. Braichev, "Sharp bounds for the type of an entire function of order less than 1 whose zeros are located on a ray and have given averaged densities", Dokl. Math., 86:1 (2012), 559-561.

[9] А. Ю. Попов, "Развитие теоремы Валирона-Левина о наименьшем возможном типе целой функции с заданной верхней $\rho$-плотностью корней”, Трудъ Крымской осенней математической школь-симпозиума, СМФН, 49, РУДН, М., 2013, 132-164; англ. пер.: А. Yu. Popov, "Development of the Valiron-Levin theorem on the least possible type of entire functions with a given upper $\rho$-density of roots", J. Math. Sci. (N. Y.), 211:4 (2015), 579-616.

[10] В. Б. Шерстюков, "Минимальное значение типа целой функции порядка меньше единицы с нулями заданных плотностей, лежащими в угле", Материалы 17-й международной Саратовской зимней школы "Современные проблемы теории функций и их приложения”, Изд-во Саратовского ун-та, Саратов, 2014, 308-310.

[11] Г. Г. Брайчев, "Точные оценки типов целых функций с нулями на лучах", Maтем. заметки, 97:4 (2015), 503-515; англ. пер.: G. G. Braichev, "Sharp estimates of types of entire functions with zeros on rays", Math. Notes, 97:4 (2015), 510-520.

[12] А. П. Прудников, Ю. А. Брычков, О. И. Маричев, Интегралы и ряды. Элементарные функиии, Наука, М., 1981, 799 с.; англ. пер.: А. P. Prudnikov, Yu. A. Brychkov, O. I. Marichev, Integrals and series, v. 1: Elementary functions, Gordon \& Breach, New York, 1986, 798 pp.

[13] Г. Г. Брайчев, Введение в теорию роста выпуклых и целых функиий, Прометей, M., 2005, 232 c.

[14] N. H. Bingham, C. M. Goldie, J.L. Teugels, Regular variation, Encyclopedia Math. Appl., 27, Cambridge Univ. Press, Cambridge, 1987, xx+491 pp.

[15] R. P. Boas, Jr., Entire functions, Academic Press, New York, 1954, x+276 pp.

\section{Георгий Генрихович Брайчев (Georgiy G. Braichev)}

Московский педагогический государственный университет

E-mail: braichev@mail.ru
Поступила в редакцию 01.02.2015 и 07.08.2015 